\title{
General structure of Thomas-Whitehead gravity
}

\author{
Samuel Brensinger॰* \\ Department of Mathematics, The University of Dayton, Dayton, Ohio 45469, USA \\ Kenneth Heitritter $\odot^{\dagger}$ and Vincent G. J. Rodgers $\odot^{\ddagger}$ \\ Department of Physics and Astronomy, The University of Iowa, Iowa City, Iowa 52242, USA \\ Kory Stiffler $\circledast^{\S}$ \\ Brown Theoretical Physics Center and Department of Physics, Brown University, \\ Providence, Rhode Island 02912-1843, USA
}

(Received 16 September 2020; accepted 21 January 2021; published 25 February 2021)

\begin{abstract}
Thomas-Whitehead (TW) gravity is a projectively invariant model of gravity over a d-dimensional manifold that is intimately related to string theory through reparametrization invariance. Unparametrized geodesics are the ubiquitous structure that ties together string theory and higher dimensional gravitation. This is realized through the projective geometry of Tracy Thomas. The projective connection, due to Thomas and later Whitehead, admits a component that in one dimension is in one-to-one correspondence with the coadjoint elements of the Virasoro algebra. This component is called the diffeomorphism field $\mathcal{D}_{a b}$ in the literature. It also has been shown that in four dimensions, the TW action collapses to the EinsteinHilbert action with cosmological constant when $\mathcal{D}_{a b}$ is proportional to the Einstein metric. These previous results have been restricted to either particular metrics, such as the Polyakov 2D metric, or were restricted to coordinates that were volume preserving. In this paper, we review TW gravity and derive the gauge invariant TW action that is explicitly projectively invariant and general coordinate invariant. We derive the covariant field equations for the TW action and show how fermionic fields couple to the gauge invariant theory. The independent fields are the metric tensor $g_{a b}$, the fundamental projective invariant $\Pi_{b c}^{a}$, and the diffeomorphism field $\mathcal{D}_{a b}$.
\end{abstract}

DOI: 10.1103/PhysRevD.103.044060

\section{INTRODUCTION}

The geometric classification of manifolds via their geodesics as opposed to distances between points (metrical) is an old notion. Indeed in his inaugural professorial lecture at Cambridge University in 1863, Cayley remarked that "descriptive geometry includes metrical geometry" and "descriptive geometry is in fact all geometry" [1]. In this reference, descriptive geometry corresponds to projective geometry. The question of whether the family of geodesics could uniquely determine the metric for general relativity was investigated by Cartan in [2,3] and further developed

\footnotetext{
*sbrensinger1@udayton.edu

${ }^{\dagger}$ kenneth-heitritter@uiowa.edu

*vincent-rodgers@uiowa.edu

${ }^{\S}$ kory_stiffler@brown.edu; Also at Department of Physics and Astronomy, The University of Iowa, Iowa City, Iowa 52242, USA.

Published by the American Physical Society under the terms of the Creative Commons Attribution 4.0 International license. Further distribution of this work must maintain attribution to the author(s) and the published article's title, journal citation, and DOI. Funded by SCOAP ${ }^{3}$.
}

by Thomas [4,5] and Whitehead [6]. The answer is that connections can only be determined up to equivalences classes. A resurgence of investigations into the physical ramifications of metrical versus descriptive (projective) geometry can be found in the literature [7-11]. On the other hand, the Virasoro algebra [12] is considered to be at the heart of string theory. It is usually viewed through its relationship with conformal symmetry, where two copies of the Virasoro algebra define the conformal algebra. However, the relationship between string theory and the Virasoro algebra also has an even more primitive origin through its identity as a one-dimensional vector space [13] and projective structure $[2,14,15]$. Since the coadjoint orbits admit a natural symplectic structure, their geometric actions provide an avenue to the two-dimensional field theories that can be associated with quantum gravity [16-18]. Furthermore, when married with an affine Lie algebra (a Kac-Moody algebra), one finds that the coadjoint elements appear as background sources for the twodimensional gravitation (Virasoro sector) and gauge (Kac-Moody sector) theories. The background fields in the Kac-Moody sector correspond to the vector potentials which serve as the gauge connections, $A_{a}$, for Yang-Mills 
theories. It was suggested in [19] that the coadjoint elements of the Virasoro sector also could be put on an equivalent footing with the Kac-Moody sector if the coadjoint elements of the Virasoro algebra could also have an associated "gauge" field in higher dimensions. The posited field was dubbed the diffeomorphism field, $\mathcal{D}_{a b}$. This realization was recently established in [20], when Kirillov's observation $[14,15]$ that the coadjoint elements of the Virasoro algebra are in one-to-one correspondence with Sturm-Liouville $[21,22]$ operators was reexamined. The authors were able to use the one-dimensional projective structure to provide a bridge between the Virasoro algebra and projective geometry in higher dimensions. Thus the analogous "gauge" symmetry due to reparametrization invariance in the Virasoro sector is projective invariance and the diffeomorphism field corresponds to projective connections. With this, the diffeomorphism field that appears in two dimensions through the geometric action as a background field has a different interpretation than that of expectation values of external energy-momentum tensors, as in conformal field theories. Furthermore the diffeomorphism field can acquire dynamics as a fundamental field through the projective curvature squared terms. Some of the entangled relationship between conformal geometry and projective geometry has been studied in [11,23-27]. For a good review see [28].

So far, discussions of dynamical projective connections $[20,29]$ have been restricted to particular metrics that are focused on the 2D Polyakov metric [30,31] or Einstein geometries in four dimensions where compatibility has been enforced. In this paper we generalize those considerations for any space-time dimensions and exhibit a Lagrangian that is explicitly projectively invariant and general coordinate invariant, i.e., gauge invariant. We will briefly review the salient features of the study of geodesics through the Thomas-Whitehead connection, the Thomas cone and tensor and fermion representations on the Thomas cone. Then, by using the Palatini [32] formalism, we explicitly construct the gauge invariant ThomasWhitehead gravitational action (TW) [20], the gauge invariant Dirac action and covariant field equations, its coupling to arbitrary Yang-Mills theories, and the energymomentum tensor. This work can be extended to include higher-order interactions, using the projective version of Lovelock gravity [33] to classically maintain an initial value formulation. We will conclude with remarks on geodesic deviations as it is there that contributions through gravitational radiation may become manifest.

\section{FROM GEODESICS TO PROJECTIVE CURVATURE}

In its most pragmatic form, string theory can be thought of as regulating the Feynman diagrams in gravitational theories by adding a small spacelike curve to the point particle. This activity already endows the string with a projective structure. The curves are parametrized by vector fields, say $\zeta^{a}=\frac{d x^{a}}{d \sigma}$, which allows one to take the intrinsic or absolute derivative of any vector field along these curves. In one dimension the Virasoro algebra is the algebra of centrally extended vector fields on a line or circle and a projective structure emerges $[2,3,15]$.

\section{A. Geodetics}

In any dimension, the intrinsic (or absolute) derivative of a vector field $v^{a}$ along a curve $\mathcal{C}$ parametrized by $\sigma$ is given by

$$
\frac{D v^{a}}{d \sigma} \equiv \frac{d v^{a}}{d \sigma}+\Gamma_{b c}^{a} v^{b} \zeta^{c},
$$

where $\Gamma_{b c}^{a}$ are connection coefficients associated with a connection $\nabla_{a}$ and $\zeta^{a}$ is the tangent vector $\frac{d x^{a}}{d \sigma}$ along the curve $\mathcal{C}$. The connection is assumed to be torsion-free and therefore satisfies the symmetry relation $\Gamma_{b c}^{a}=\Gamma_{c b}^{a}$. An affine geodesic generalizes the notion of a straight line and $\zeta^{a}$ is said to be geodesic if the change of $\zeta^{a}$ along the curve $\mathcal{C}$ parametrized by $\sigma$ is to be proportional to itself, i.e.,

$$
\frac{D \zeta^{a}}{d \sigma}=f(\sigma) \zeta^{a},
$$

where $f(\sigma)$ is the proportionality function. This yields the affine geodesic equation,

$$
\frac{d^{2} x^{a}}{d \sigma^{2}}+\Gamma_{b c}^{a} \frac{d x^{b}}{d \sigma} \frac{d x^{c}}{d \sigma}=f(\sigma) \frac{d x^{a}}{d \sigma} .
$$

One may change the parametrization from $\sigma$ to $u(\sigma)$ by writing

$$
\frac{d}{d u}=\frac{d \sigma}{d u} \frac{d}{d \sigma}
$$

and for a suitable choice $u(\sigma)$ we can eliminate the righthand side of Eq. (3) to write the geodetic equation

$$
\frac{d^{2} x^{a}}{d u^{2}}+\Gamma_{b c}^{a} \frac{d x^{b}}{d u} \frac{d x^{c}}{d u}=0
$$

Here the parameter $u$ is said to be an affine parameter with respect to the connection $\nabla_{a}$ as

$$
\frac{d x^{b}}{d u} \nabla_{b} u=1
$$

Although the parametrization may have changed, the curves remain the same. Furthermore different connections, say $\hat{\nabla}_{a}$ and $\nabla_{a}$ can sometimes admit the same geodesics. If so, then $\hat{\nabla}$ and $\nabla$ belong to the same projective equivalence class. Thomas showed how one can write a gauge theory 
over this projective symmetry $[4,5]$. We discuss this gauge theory presently.

\section{B. Projectively equivalent paths}

Consider a d-dimensional manifold $\mathcal{M}$ with coordinates $x^{a}$ where italic latin indices $a, b, c, m, n, \ldots=$ $0,1, \ldots, \mathrm{d}-1$. Let $\hat{\nabla}_{a}$ be a connection on $\mathcal{M}$ where $\zeta^{a}$ is geodetic, i.e.,

$$
\zeta^{b} \hat{\nabla}_{b} \zeta^{a}=\frac{d^{2} x^{a}}{d \tau^{2}}+\hat{\Gamma}^{a}{ }_{b c} \frac{d x^{b}}{d \tau} \frac{d x^{c}}{d \tau}=0 .
$$

Now consider another connection whose coefficients are defined as

$$
\Gamma_{b c}^{a}=\hat{\Gamma}_{b c}^{a}+\delta^{a}{ }_{b} v_{c}+\delta^{a}{ }_{c} v_{b},
$$

where $v_{b}$ is an arbitrary one-form. The geodesic equation for this connection is then

$$
\zeta^{b} \nabla_{b} \zeta^{a}=\frac{d^{2} x^{a}}{d \tau^{2}}+\Gamma_{b c}^{a} \frac{d x^{b}}{d \tau} \frac{d x^{c}}{d \tau}=f(\tau) \frac{d x^{a}}{d \tau},
$$

and where $f(\tau)=2 v_{b} \frac{d x^{b}}{d \tau}$. Since Eq. (8) can also be made geodetic by a suitable reparametrization of $\tau$ to $u(\tau)$ both Eqs. (6) and (8) admit the same geodesic curves. Equation (7) is called a projective transformation and establishes the projective equivalence relation, $\hat{\Gamma}_{b c}^{a} \sim \Gamma^{a}{ }_{b c}$.

In $[4,5]$, Thomas presents a "gauge" theory of projectively equivalent connections that is projectively invariant and general coordinate invariant. This begins by defining the fundamental projective invariant $\Pi^{a}{ }_{b c}$

$$
\Pi_{b c}^{a} \equiv \Gamma_{b c}^{a}-\frac{1}{(\mathrm{~d}+1)} \delta_{(b}^{(b} \Gamma_{c) m}^{m}
$$

which is traceless by construction

$$
\Pi^{a}{ }_{b a}=\Pi_{a b}^{a}=0
$$

and invariant under a projective transformation, Eq. (7), for an arbitrary one form $v_{a}$. Using the fundamental projective invariant $\Pi_{b c}^{a}$ one can write a geodetic equation

$$
\frac{d^{2} x^{a}}{d \tau^{2}}+\Pi_{b c}^{a} \frac{d x^{b}}{d \tau} \frac{d x^{c}}{d \tau}=0
$$

that is projectively invariant. However this equation is not covariant as $\Pi_{b c}^{a}$ transforms as

$$
\begin{aligned}
\Pi_{b c}^{\prime a}= & J_{f}^{a}\left(\Pi_{d e}^{f} \overline{\boldsymbol{J}}_{b}{ }_{b} \overline{\boldsymbol{J}}_{c}{ }_{c}+\frac{\partial^{2} x^{f}}{\partial x^{\prime b} \partial x^{\prime c}}\right) \\
& +\frac{1}{d+1} \frac{\partial \log |J|}{\partial x^{d}}\left(\overline{\boldsymbol{J}}_{b}{ }_{b} \delta^{a}{ }_{c}+\overline{\boldsymbol{J}}^{d}{ }_{c} \delta^{a}{ }_{b}\right)
\end{aligned}
$$

under a general coordinate transformation from $x \rightarrow x^{\prime}(x)$ with $J_{b}^{a}=\frac{\partial x^{a}}{\partial x^{b}}$, the Jacobian of the transformation. We will denote the inverse Jacobian as $\bar{J}^{a}{ }_{b}=\frac{\partial x^{a}}{\partial x^{\prime b}}$. The last summand spoils the covariance and can be related to volume, as it involves the determinant of the Jacobian of the transformation $J=\operatorname{det}\left(J_{b}^{a}\right)$. Thomas then constructs a line bundle over $\mathcal{M}$ which is a $\mathrm{d}+1$-dimensional manifold $\mathcal{N}$ referred to as the Thomas cone $[34,36]$. The coordinates on the Thomas cone are $\left(x^{0}, x^{1}, \ldots, x^{\mathrm{d}-1}, \lambda\right)$, where $\lambda$ is denoted the volume coordinate. Since the volume coordinate, $\lambda$, takes values $0<\lambda<\infty, \mathcal{N}$ is called a cone. The coordinates transform as

$x^{\prime \alpha}=\left(x^{\prime 0}\left(x^{d}\right), x^{\prime 1}\left(x^{d}\right), \ldots, x^{\prime \mathrm{d}-1}\left(x^{d}\right), \lambda^{\prime}=\lambda|J|^{-\frac{1}{\mathrm{~d}+1}}\right)$.

From here on, we refer to transformations in Eq. (13) as TC $\mathcal{N}$-transformations. Here, Greek indices are over $\mathcal{N}$ coordinates and take values $\alpha, \beta, \mu, \ldots=0,1,2, \ldots, \mathrm{d}$ and italic latin indices are over coordinates on $\mathcal{M}$ and take values $a, b, m, n, \ldots=0,1,2, \ldots \mathrm{d}-1$. We reserve the index $\lambda$ and the upright letter $\mathrm{d}$ to refer to the volume coordinate $x^{\mathrm{d}}=x^{\lambda}=\lambda$. For every coordinate transformation on $\mathcal{M}$ there is a unique coordinate transformation on $\mathcal{N}$.

\section{Thomas projective connections}

Thomas was able to find a connection on $\mathcal{N}$ that transforms as a connection by extending the fundamental projective invariant to a $\mathrm{d}+1$-dimensional projective connection $\tilde{\Pi}^{\alpha}{ }_{\mu \nu}$. It is defined as follows [4,6,37]:

$$
\begin{aligned}
& \tilde{\Pi}_{\lambda \beta}^{\alpha}=\tilde{\Pi}_{\beta \lambda}^{\alpha}=-\frac{1}{\mathrm{~d}+1} \delta_{\beta}^{\alpha}, \\
& \tilde{\Pi}_{b c}^{a}=\tilde{\Pi}_{c b}^{a}=\Pi_{b c}^{a}, \\
& \tilde{\Pi}_{a b}^{\lambda}=\tilde{\Pi}_{b a}^{\lambda}=-\frac{\mathrm{d}+1}{\mathrm{~d}-1} \mathcal{R}_{a b},
\end{aligned}
$$

where $\mathcal{R}_{a b}$ is constructed from the equi-projective curvature "tensor" $\mathcal{R}^{m}{ }_{a b n}$

$$
\mathcal{R}^{m}{ }_{a b n}=\Pi^{m}{ }_{a[n, b]}+\Pi^{p}{ }_{a[n} \Pi_{b] p}^{m},
$$

with an associated equi-projective Ricci "tensor"

$$
\mathcal{R}_{a b}=\mathcal{R}^{m}{ }_{a m b} .
$$

With this, $\tilde{\Pi}^{\alpha}{ }_{\mu \nu}$ transforms as a connection under a TCNtransformation as

$$
\tilde{\Pi}^{\prime \alpha}{ }_{\mu \nu}=\frac{\partial x^{\prime \alpha}}{\partial x^{\rho}} \frac{\partial x^{\sigma}}{\partial x^{\prime \mu}} \frac{\partial x^{\beta}}{\partial x^{\prime \nu}} \tilde{\Pi}^{\rho}{ }_{\sigma \beta}+\frac{\partial^{2} x^{\beta}}{\partial x^{\prime \mu} \partial^{\prime \nu}} \frac{\partial x^{\prime \alpha}}{\partial x^{\beta}}
$$


so that one may construct a projective curvature tensor

$$
\tilde{\mathcal{R}}_{\mu \nu \beta}^{\alpha}=\tilde{\Pi}_{\mu[\beta, \nu]}^{\alpha}+\tilde{\Pi}_{\mu[\beta}{ }_{\mu[\beta} \tilde{\Pi}_{\nu] \rho}^{\alpha},
$$

whose nonvanishing components are

$$
\begin{gathered}
\tilde{\mathcal{R}}^{\lambda}{ }_{a b n}=\frac{\mathrm{d}+1}{\mathrm{~d}-1}\left(\mathcal{R}_{a[b, n]}+\Pi^{m}{ }_{a[b} \mathcal{R}_{n] m}\right), \\
\tilde{\mathcal{R}}^{m}{ }_{a b n}=\mathcal{R}^{m}{ }_{a b n}-\frac{1}{\mathrm{~d}-1} \delta_{[n}{ }^{m} \mathcal{R}_{b] a} .
\end{gathered}
$$

The projective Ricci tensor is defined as the trace of the projective curvature tensor and vanishes identically

$$
\tilde{\mathcal{R}}_{\alpha \beta} \equiv \tilde{\mathcal{R}}_{\alpha \mu \beta}^{\mu}=0
$$

This construction is only a specific example of a projective connection but it laid the ground work for the more general setting we now present.

\section{THOMAS-WHITEHEAD PROJECTIVE GEOMETRY}

\section{A. The general projective connection}

The original Thomas projective connection, $\tilde{\Pi}^{\alpha}{ }_{\mu \nu}$, can be generalized to a connection $\tilde{\Gamma}_{\beta \gamma}^{\alpha}[6,35,37]$, where explicitly

$$
\tilde{\Gamma}_{\beta \gamma}^{\alpha}=\left\{\begin{array}{l}
\tilde{\Gamma}_{\lambda a}^{\lambda}=\tilde{\Gamma}_{a \lambda}^{\lambda}=0 \\
\tilde{\Gamma}_{\lambda \lambda}^{\alpha}=0 \\
\tilde{\Gamma}_{\lambda b}^{a}=\tilde{\Gamma}_{b \lambda}^{a}=\alpha_{\lambda} \delta^{a}{ }_{b} \\
\tilde{\Gamma}_{b c}^{a}=\Pi^{a}{ }_{b c} \\
\tilde{\Gamma}_{a b}^{\lambda}=\Upsilon^{\lambda} \mathcal{D}_{a b}
\end{array}\right.
$$

and where

$$
\begin{aligned}
\Pi_{b c}^{a} & =\Gamma_{b c}^{a}+\delta_{(c}^{a} \alpha_{b)}, \\
\alpha_{a} & =-\frac{1}{\mathrm{~d}+1} \Gamma_{a m}^{m}, \\
\Upsilon^{\alpha} & =(0,0, \ldots, 0, \lambda), \\
\alpha_{\alpha} & =\left(\alpha_{a}, \lambda^{-1}\right) .
\end{aligned}
$$

Here the connection $\Gamma_{b c}^{a}$ is any representative member of the equivalence class $\left[\Gamma_{b c}^{a}\right]$ of projectively equivalent connections, related via Eq. (7), and $\alpha_{a}$ is that chosen member's trace component. However, keep in mind that $\Pi^{a}{ }_{b c}$ exists in its own right in that it is traceless and transforms like a traceless part of an affine connection. Notice also that only the $\lambda$ component for $\alpha_{\mu}$ appears in the projective connection $\tilde{\Gamma}_{\alpha \beta}^{\mu}$. On $\mathcal{M}$, the transformation laws are

$$
\begin{gathered}
\Gamma_{m n}^{a}=\frac{\partial x^{\prime a}}{\partial x^{b}} \frac{\partial x^{p}}{\partial x^{\prime m}} \frac{\partial x^{q}}{\partial x^{\prime n}} \Gamma_{p q}^{b}+\frac{\partial^{2} x^{b}}{\partial x^{\prime m} \partial^{\prime n}} \frac{\partial x^{\prime a}}{\partial x^{b}}, \\
\alpha_{a}^{\prime}=\frac{\partial x^{m}}{\partial x^{\prime a}} \alpha_{m}+\frac{\partial \log |J| \frac{1}{d+1}}{\partial x^{\prime a}} .
\end{gathered}
$$

In the above, $\mathcal{D}_{a b}$ generalizes the work of Thomas and transforms in such a way that $\tilde{\Gamma}_{\beta \gamma}^{\alpha}$ transforms as an affine connection on $\mathcal{N}$. This is the origin of the diffeomorphism field $\mathcal{D}_{a b}$. In this construction, $\Upsilon$ is the fundamental vector on the Thomas cone and satisfies the compatibility relation

$$
\tilde{\nabla}_{\alpha} \Upsilon^{\beta}=\delta_{\alpha}^{\beta}
$$

so $\Upsilon^{\beta}$ satisfies the fundamental geodesic equation with unit proportionality

$$
\Upsilon^{\beta} \tilde{\nabla}_{\beta} \Upsilon^{\alpha}=\Upsilon^{\alpha}
$$

For functions on $\mathcal{N}$

$$
\Upsilon^{\beta} \tilde{\nabla}_{\beta} f=\lambda \partial_{\lambda} f
$$

showing that $\Upsilon$ generates scaling in the $\lambda$ direction. Oneforms $\beta_{\alpha}$ on $\mathcal{N}$ are uniquely defined by $\beta_{a}$ on $\mathcal{M}$ when $\beta_{\alpha} \Upsilon^{\alpha}=1$ and the Lie derivative with respect to $\Upsilon$ vanishes i.e., $\mathcal{L}_{\Upsilon} \beta_{\rho}=0$, so that it is scale invariant. Under a TCN $\mathcal{N}$ transformation, Eq. (13), $\Upsilon^{\alpha}$ and the covariant derivative transform as

$$
\begin{aligned}
& \Upsilon^{\prime \alpha}=\frac{\partial x^{\prime \alpha}}{\partial x^{\beta}} \Upsilon^{\beta}, \\
& \nabla_{\alpha}^{\prime}=\frac{\partial x^{\beta}}{\partial x^{\prime \alpha}} \nabla_{\beta} .
\end{aligned}
$$

Demanding that $\tilde{\Gamma}_{\mu \nu}^{\alpha}$ transforms as an affine connection

$$
\tilde{\Gamma}^{\prime \alpha}{ }_{\mu \nu}=\frac{\partial x^{\prime \alpha}}{\partial x^{\rho}} \frac{\partial x^{\sigma}}{\partial x^{\prime \mu}} \frac{\partial x^{\beta}}{\partial x^{\prime \nu}} \tilde{\Gamma}_{\sigma \beta}^{\rho}+\frac{\partial^{2} x^{\beta}}{\partial x^{\prime \mu} \partial^{\prime \nu}} \frac{\partial x^{\prime \alpha}}{\partial x^{\beta}},
$$

and using the transformation laws of $\Upsilon^{\alpha}$ and $\tilde{\Gamma}^{\lambda}{ }_{a b}$, one finds that $\mathcal{D}_{a b}$ transforms under a coordinate transformation on $\mathcal{M}$ as

$$
\mathcal{D}_{a b}^{\prime}=\frac{\partial x^{m}}{\partial x^{\prime a}} \frac{\partial x^{n}}{\partial x^{\prime b}}\left(\mathcal{D}_{m n}-\partial_{m} j_{n}-j_{m} j_{n}+j_{c} \Pi^{c}{ }_{m n}\right),
$$

where we define $j_{a}=\partial_{a} \log |J|^{-\frac{1}{d+1}}$. One can show that the coordinate transformation law of $\mathcal{D}_{a b}$ as stated by Eq. (35) is an action of the general linear group on the components of $\mathcal{D}$. This property holds despite the presence of the coordinate-dependent object $\Pi_{b c}^{a}$ in the transformation law [38]. This transformation law will become important later 
in the correspondence with coadjoint elements of the Virasoro algebra in one dimension.

A general tensor on $\mathcal{M}$ with $m$-contravariant and $n$-covariant indices we express as

$$
T_{b(n)}^{a(m)}=T^{a_{1} a_{2} \ldots a_{m}} b_{1} b_{2} \ldots b_{n} .
$$

In what follows we refer to $(m, n)$-tensors on $\mathcal{M}$ as objects that transform as

$$
T_{b(n)}^{\prime a(m)}=\frac{\partial x^{\prime a_{1}}}{\partial x^{p_{1}}} \ldots \frac{\partial x^{\prime a_{m}}}{\partial x^{p_{m}}} \frac{\partial x^{q_{1}}}{\partial x^{\prime b_{1}}} \ldots \frac{\partial x^{q_{n}}}{\partial x^{\prime b_{n}}} T_{q(n)}^{p(m)}
$$

under coordinate transformations. Similarly, we refer to objects as $(m, n)$-TC tensors on $\mathcal{N}$ that transform as

$T_{\beta(n)}^{\prime \alpha(m)}=\frac{\partial x^{\prime \alpha_{1}}}{\partial x^{\mu_{1}}} \ldots \frac{\partial x^{\prime \alpha_{m}}}{\partial x^{\mu_{m}}} \frac{\partial x^{\nu_{1}}}{\partial x^{\prime \beta_{1}}} \ldots \frac{\partial x^{\nu_{n}}}{\partial x^{\prime \beta_{n}}} T_{\nu(n)}^{\mu(m)}$

under a TCN-transformation. This will allow us to build actions that are invariant with respect to $\mathrm{TCN}$ transformations.

\section{B. Geodetics revisited}

Before discussing projective curvature relations, we now revisit geodesics and geodetics to illuminate the projective connection. Consider a geodetic on $\mathcal{N}$ associated with the vector field $\zeta^{\alpha}=\frac{d x^{\alpha}}{d u}$. The parameter $u$ is an affine parameter for $\tilde{\nabla}$ such that

$$
\zeta^{\alpha} \tilde{\nabla}_{\alpha} \zeta^{\beta}=0
$$

Separating the $\mathcal{M}$ coordinates from $\lambda$, we have the expressions

$$
\begin{gathered}
\frac{d^{2} x^{a}}{d u^{2}}+\Pi_{b c}^{a} \frac{d x^{b}}{d u} \frac{d x^{c}}{d u}=-2 \frac{1}{\lambda}\left(\frac{d \lambda}{d u}\right) \frac{d x^{a}}{d u}, \\
\frac{d^{2} \lambda}{d u^{2}}+\lambda \mathcal{D}_{b c} \frac{d x^{b}}{d u} \frac{d x^{c}}{d u}=0 .
\end{gathered}
$$

Together, these equations are covariant and projectively invariant. Let us consider a reparametrization that can render Eq. (40) geodetic. In other words, does there exist a parameter $\tau$ that is affine with respect to the projective invariant $\Pi_{b c}^{a}$ ? Let $u \rightarrow \tau(u)$ so that

$$
\frac{d^{2} \tau}{d u^{2}}=-2\left(\frac{1}{\lambda} \frac{d \lambda}{d u}\right) \frac{d \tau}{d u}
$$

This will eliminate the right-hand side of Eq. (40) and we can use this to eliminate $\lambda$ in Eq. (41) with

$$
\frac{d^{2} \lambda}{d u^{2}}=\frac{\lambda}{4} \cdot \frac{3\left(\frac{d^{2} \tau}{d u^{2}}\right)^{2}-2\left(\frac{d^{3} \tau}{d u^{3}}\right) \frac{d \tau}{d u}}{\left(\frac{d \tau}{d u}\right)^{2}}
$$

With this, one finds that the reparametrization is viable if

$$
\mathcal{D}_{b c} \frac{d x^{b}}{d u} \frac{d x^{c}}{d u}=\frac{1}{2} \cdot \frac{\frac{d \tau}{d u}\left(\frac{d^{3} \tau}{d u^{3}}\right)-\frac{3}{2}\left(\frac{d^{2} \tau}{d u^{2}}\right)^{2}}{\left(\frac{d \tau}{d u}\right)^{2}} \equiv \frac{1}{2} S(\tau: u),
$$

where $S(\tau: u)$ is the Schwarzian derivative of $\tau$ with respect to $u$. For example, if the kinetic term $\mathcal{D}_{b c} \frac{d x^{b}}{d u} \frac{d x^{c}}{d u}$ vanishes, then requisite reparametrizations that render $\tau$ affine are the Möbius transformations $\tau=\frac{a u+b}{c u+d}$, where $a, b, c$, and $d$ are real numbers. Another familiar example is when $\mathcal{D}_{b c} \frac{d x^{b}}{d u} \frac{d x^{c}}{d u}=\frac{m^{2}-1}{2 u^{2}}$ and the requisite transformations are the exponential Möbius transformations $\tau=\left(\frac{a u^{m}+b}{c u^{m}+d}\right)$. This corresponds to the coadjoint orbits of the Virasoro algebra denoted by $\operatorname{Diff}\left(S^{1}\right) / \operatorname{SL}(2, m)$, where the isotropy group is generated by $L_{m}, L_{0}, L_{-m}$. A Möbius transformation is a one-dimensional projective transformation, so we see that the preferred class of parameters for $\Pi_{b c}^{a}$ is preserved by projective transformations rather than affine transformations. This motivates the description of $\Pi_{b c}^{a}$ as a projective connection. The inclusion of $\Pi_{b c}^{a}$ in the TW connection, which incorporates the field $\mathcal{D}_{b c}$, allows us to apply techniques that are typically available for affine connections.

\section{Projective geometry}

One constructs the projective curvature tensor in the usual way

$$
\begin{gathered}
{\left[\tilde{\nabla}_{\alpha}, \tilde{\nabla}_{\beta}\right] V^{\gamma}=\mathcal{K}_{\rho \alpha \beta}^{\gamma} V^{\rho},} \\
{\left[\tilde{\nabla}_{\alpha}, \tilde{\nabla}_{\beta}\right] V_{\gamma}=-\mathcal{K}_{\gamma \alpha \beta}^{\rho} V_{\rho},}
\end{gathered}
$$

from connections that transform as in Eq. (34). In terms of the connections, the curvature can be written explicitly as

$$
\mathcal{K}_{\mu \nu \beta}^{\alpha}=\tilde{\Gamma}_{\mu[\beta, \nu]}^{\alpha}+\tilde{\Gamma}_{\mu[\beta}^{\rho} \tilde{\Gamma}_{\nu] \rho}^{\alpha} .
$$

This transforms as a $(1,3)$ TC tensor on $\mathcal{N}$. Using Eq. (22) to expand $\tilde{\Gamma}_{\mu \nu}^{\alpha}$ we find the only nonvanishing components of the projective curvature tensor to be

$$
\begin{gathered}
\mathcal{K}^{a}{ }_{b c d}=\mathcal{R}_{b c d}^{a}+\delta^{a}{ }_{[c} \mathcal{D}_{d] b}, \\
\mathcal{K}^{\lambda}{ }_{c a b}=\lambda \partial_{[a} \mathcal{D}_{b] c}+\lambda \Pi^{d}{ }_{c[b} \mathcal{D}_{a] d} .
\end{gathered}
$$

We will also find it useful later on to have a $\lambda$-independent version of $\mathcal{K}_{c a b}^{\lambda}$. We define this symbol as 


$$
\breve{\mathcal{K}}_{c a b}^{\lambda} \equiv \frac{1}{\lambda} \mathcal{K}_{c a b}^{\lambda}=\partial_{[a} \mathcal{D}_{b] c}+\Pi_{c[b}^{d} \mathcal{D}_{a] d}
$$

By contracting the first and third indices of the projective curvature tensor, we can write the projective Ricci tensor whose only nonvanishing components are

$$
\mathcal{K}_{b d}=\mathcal{R}_{b d}+(\mathrm{d}-1) \mathcal{D}_{b d}
$$

$\mathcal{R}_{b d}$ is the equi-projective Ricci tensor from Eq. (16). The expressions in Eq. (48) are precisely of the form seen in conformal geometry

$$
\begin{aligned}
R_{b c d}^{a} & =W_{b c d}^{a}+\delta^{a}{ }_{[c} P_{d] b}, \\
C_{c a b} & =\partial_{[a} P_{b] c}+\Gamma_{c[b}^{d} P_{a] d},
\end{aligned}
$$

where $W^{a}{ }_{b c d}$ is the Weyl tensor, $P_{d b}$ is the Schouten tensor, and $C_{c a b}$ is the Cotton-York tensor. In the above, $W^{a}{ }_{b c d}$ is analogous to $\mathcal{K}^{a}{ }_{b c d}$ in Eq. (48). If we consider the contraction of the projective curvature tensor with a volume one-form $g_{\mu}$, that transforms as Eq. (28) and is also invariant under projective transformations, we can form the projective Cotton-York tensor, $\mathcal{K}(g)_{\nu \alpha \beta} \equiv g_{\mu} \mathcal{K}^{\mu}{ }_{\nu \alpha \beta}$. Then we can write

$$
\begin{aligned}
\mathcal{K}(g)_{n a b} & =g_{\mu} \mathcal{K}^{\mu}{ }_{n a b} \\
& =\mathcal{P}_{[b|n| ; a]}-\Delta_{n} \mathcal{P}_{[a b]}+\Delta_{[a} \mathcal{P}_{b] n}+R^{m}{ }_{n a b} \Delta_{m}
\end{aligned}
$$

where $\Delta_{a} \equiv g_{a}-\alpha_{a}$ is a one-form on $\mathcal{M}$. $\mathcal{K}(g)_{\nu \alpha \beta}$ is now explicitly seen as a $(0,3)$-TC tensor on $\mathcal{N}$ and $\mathcal{K}_{\text {nab }}$ is a $(0,3)$-tensor on $\mathcal{M}$. When we introduce a metric tensor $g_{a m}$ on $\mathcal{M}$ in the next section, we will find that $g_{\mu}=\left(g_{a}, \frac{1}{\lambda}\right)$, where $g_{a} \equiv-\frac{1}{\mathrm{~d}+1} \partial_{a} \log \sqrt{|g|}$ is a suitable volume oneform, Eq. (60). This also introduces the projective Schouten tensor [26] $\mathcal{P}_{a b}$, which is a $(0,2)$-tensor on $\mathcal{M}$. The form of $\tilde{\Gamma}^{\alpha}{ }_{\mu \nu}$ in Eq. (22) allows for $\mathcal{D}_{a b}$ to become dynamical as $\mathcal{K}^{\alpha}{ }_{\mu \alpha \beta} \neq 0$, relaxing the Ricci flat condition in $[4,5,35]$. This allows us to extend the Einstein-Hilbert action to projective geometry as in [20,29].

If we choose a member of the equivalence class $\left[\Gamma^{c}{ }_{a b}\right]$, then we may express $\Pi^{c}$ ab in terms of a specific connection and its associated trace $\alpha_{\mu}$. With this, one may write $\mathcal{P}_{a b}$ in terms of $\mathcal{D}_{a b}$ as

$$
\mathcal{P}_{b c}=\mathcal{D}_{b c}-\partial_{b} \alpha_{c}+\Gamma_{b c}^{e} \alpha_{e}+\alpha_{b} \alpha_{c}
$$

The above is a generalization of [20,29], where constant volume coordinates were used and $\Gamma_{b c}^{e}$ was regarded as Levi-Civita so $\alpha_{a}=0$. Then, in that case, $\mathcal{D}_{a b}=\mathcal{P}_{a b}$ and is a tensor in the volume preserving coordinates. As stated above, $\mathcal{P}_{a b}$ transforms as a tensor on $\mathcal{M}$

$$
\mathcal{P}_{a b}^{\prime}=\frac{\partial x^{m}}{\partial x^{\prime a}} \frac{\partial x^{n}}{\partial x^{\prime n}} \mathcal{P}_{m n}
$$

which we may call the projective Schouten tensor in analogy with conformal geometry.

\section{COVARIANT METRIC TENSOR ON $\mathcal{N}$}

In projective geometry, a vector field $\chi$ on $\mathcal{M}$ may be lifted to a vector field $\tilde{\chi}$ on $\mathcal{N}$ by writing

$$
\tilde{\chi}^{\alpha} \partial_{\alpha}=-\left(\lambda \chi^{a} \kappa_{a}\right) \partial_{\lambda}+\chi^{a} \partial_{a}
$$

where $\kappa_{a}$ is some object that transforms as $j_{a}$ in Eq. (35), i.e.,

$$
\kappa_{a}^{\prime}=\frac{\partial x^{m}}{\partial x^{\prime a}} \kappa_{m}-\frac{1}{\mathrm{~d}+1} \frac{\partial \log J}{\partial x^{\prime a}}
$$

under a general coordinate transformation on $\mathcal{M}$. We write the components of $\tilde{\chi}$ as

$$
\tilde{\chi}^{\alpha}=\left(\chi^{a},-\lambda x^{b} \kappa_{b}\right)
$$

Similarly, a one-form $v$ on $\mathcal{M}$ can be related to a projective one-form $\tilde{v}$ via

$$
\tilde{v}_{\beta}=\left(v_{b}+\kappa_{b}, \frac{1}{\lambda}\right) .
$$

It is clear that $\tilde{\chi}^{\alpha} \tilde{v}_{\alpha}=\chi^{a} v_{a}$. A generic vector on $\mathcal{N}$, which has components $\eta_{\perp}$ that are unrelated to vectors on $\mathcal{M}$, may be written as

$$
\tilde{\eta}^{\beta}=\left(\eta_{\|}^{b}, \lambda\left(\eta_{\perp}-\kappa_{a} \eta_{\|}^{a}\right)\right)
$$

The fundamental vector field $\Upsilon$ in Eq. (26) has no component parallel to $\mathcal{M}$, for example.

We are interested in building an invariant action using the projective curvature. This will require a soldering metric which transforms as a tensor on $\mathcal{N}$ and which is projectively invariant. Taking a metric $g_{a b}$ on $\mathcal{M}$, one may view this soldering metric as the local tensor product of two one-forms and write

$$
G_{\mu \nu}=\left[\begin{array}{c}
g_{a b}-\lambda_{0}^{2} g_{a} g_{b}-\frac{\lambda_{0}^{2}}{\lambda} g_{a} \\
-\frac{\lambda_{0}^{2}}{\lambda} g_{b}-\frac{\lambda_{0}^{2}}{\lambda^{2}}
\end{array}\right]
$$

Here we have replaced $\kappa_{a}$ with $g_{a} \equiv-\frac{1}{\mathrm{~d}+1} \partial_{a} \log \sqrt{|g|}$ as it is naturally built from the metric degrees of freedom and does not introduce a connection. The constant $\lambda_{0}$ has units of length (like $\lambda$ ), and ensures that $G_{\mu \nu}$ remains dimensionless when $g_{a b}$ is dimensionless. Since $G_{\mu \nu}$ depends only on 
the spacetime metric $g_{a b}$, it is indeed projectively invariant. One can check that $G_{\mu \nu}$ satisfies the transformation law

$$
G^{\prime}(y)_{\mu \nu}=\frac{\partial x^{\alpha}}{\partial y^{\mu}} \frac{\partial x^{\beta}}{\partial y^{\nu}} G(x)_{\alpha \beta}
$$

when $\left(x^{a}, x^{\lambda}\right) \rightarrow\left(y^{a}, y^{\lambda}\right)=\left(y^{a}, x^{\lambda}|J|^{-\frac{1}{\mathrm{~d}+1}}\right)$. Furthermore, under this coordinate change the volume form on $\mathcal{N}$ remains invariant, i.e.,

$$
\sqrt{\left|G\left(x^{a}, x^{\lambda}\right)\right|} d x^{\lambda} d^{\mathrm{d}} x=\sqrt{\left|G\left(y^{a}, y^{\lambda}\right)\right|} d y^{\lambda} d^{\mathrm{d}} y .
$$

Here $G\left(x^{a}, x^{\lambda}\right)$ and $G\left(y^{a}, y^{\lambda}\right)$ are the metric determinants in the different coordinates. This follows since from Eq. (60), we see that

$$
|G|=|g| \cdot \frac{\lambda_{0}^{2}}{\lambda^{2}}
$$

where $g$ is the determinant of $g_{a b}$ on $M$. Since $y^{\lambda}=$ $x^{\lambda}|J|^{-\frac{1}{d+1}}$ and $\frac{1}{\lambda} \rightarrow \frac{1}{\lambda}|J|^{\frac{1}{d+1}}$, these terms exactly conspire in Eq. (62) to maintain the invariant volume on $\mathcal{N}$. Again, this motivates why $\lambda$ is called the volume coordinate. Lastly, the inverse of $G_{\mu \nu}$ is given by

$$
G^{\mu \nu}=\left[\begin{array}{c}
g^{a b}-\lambda g^{a m} g_{m} \\
-\lambda g^{b m} g_{m} \frac{\lambda^{2}}{\lambda_{0}{ }^{2}}\left(-1+g^{m n} \lambda_{0}^{2} g_{m} g_{n}\right)
\end{array}\right],
$$

where $g^{a b}$ is the inverse of the spacetime metric $g_{a b}$. This metric generalizes the work in [20,29], allowing TW gravity to be used in any coordinates. We can succinctly write the metric and its inverse as

$$
\begin{gathered}
G_{\alpha \beta}=\delta^{a}{ }_{\alpha} \delta^{b}{ }_{\beta} g_{a b}-\lambda_{0}^{2} g_{\alpha} g_{\beta}, \\
G^{\alpha \beta}=g^{a b}\left(\delta^{\alpha}{ }_{a}-g_{a} \Upsilon^{\alpha}\right)\left(\delta^{\beta}{ }_{b}-g_{b} \Upsilon^{\beta}\right)-\lambda_{0}^{-2} \Upsilon^{\alpha} \Upsilon^{\beta},
\end{gathered}
$$

where we have defined $g_{\alpha} \equiv\left(g_{a}, \frac{1}{\lambda}\right)$. In TW gravity, the metric $g_{a b}$, the projective invariant $\Pi_{b c}^{a}$, and the diffeomorphism field $\mathcal{D}_{a b}$ will be treated as independent degrees of freedom in the spirit of the Palatini formalism [32].

\section{V. $\tilde{\gamma}^{\mu}$ ON $\mathcal{N}$}

Now we seek the $\tilde{\gamma}^{\alpha}$ matrices associated with the projective metric $G_{\mu \nu}$ given by Eq. (60). The gamma matrices, $\gamma^{m}$, on a d-dimensional spacetime are defined by

$$
\left\{\gamma^{m}, \gamma^{n}\right\}=2 g^{m n} I_{N}
$$

where $\{\cdot, \cdot\}$ is the anticommutator, $g_{\mu \nu}$ is the spacetime metric, $N=2^{\lfloor\mathrm{d} / 2\rfloor}$, and $I_{N}$ is the $N \times N$ identity matrix.
Let $\tilde{\gamma}^{\mu}$ be the gamma matrices for the metric $G_{\mu \nu}$ on $\mathcal{N}$. These matrices satisfy

$$
\left\{\tilde{\gamma}^{\mu}, \tilde{\gamma}^{\nu}\right\}=2 G^{\mu \nu} I_{N}
$$

as in Eq. (67). We will stay in even space-time dimensions. In this case, the gamma matrices $\tilde{\gamma}^{\mu}$ for $G_{\mu \nu}$ will have the same dimension as the gamma matrices $\gamma^{m}$ for $g_{m n}$.

Using the inverse of $G_{\mu \nu}$, Eq. (64), we immediately must have $\tilde{\gamma}^{\mu}=\gamma^{\mu}$ if $\mu$ is a spacetime coordinate index, say $m$, and where $\gamma^{m}$ are the gamma matrices for the spacetime metric $g_{m n}$. The remaining gamma matrix is $\tilde{\gamma}^{\lambda}$. This matrix must satisfy

$$
\begin{array}{r}
\left\{\tilde{\gamma}^{\lambda}, \tilde{\gamma}^{m}\right\}=-2 \lambda g^{m n} g_{n} I_{N}, \quad m=0, \ldots, \mathrm{d}-1, \\
2\left(\tilde{\gamma}^{\lambda}\right)^{2}=\left\{\tilde{\gamma}^{\lambda}, \tilde{\gamma}^{\lambda}\right\}=2 \frac{\lambda^{2}}{\lambda_{0}{ }^{2}}\left(-1+g^{m n} \lambda_{0}^{2} g_{m} g_{n}\right) I_{N} .
\end{array}
$$

Recall the chiral matrix $\gamma^{5}$ in four-dimensional spacetime. We will refer to it as $\gamma^{\mathrm{d}+1}$ in the general even dimensional case. It satisfies

$$
\begin{gathered}
\left\{\gamma^{\mathrm{d}+1}, \gamma^{m}\right\}=0, \\
\left(\gamma^{\mathrm{d}+1}\right)^{2}=I_{N} .
\end{gathered}
$$

Comparing Eqs. (69) and (70) to Eqs. (71) and (72), we see that we should have

$$
\tilde{\gamma}^{\lambda}=-\frac{\lambda}{\lambda_{0}}\left(i \gamma^{\mathrm{d}+1}+\lambda_{0} g_{m} \gamma^{m}\right)
$$

as the final gamma matrix for $G_{\mu \nu}$. Explicitly, the chiral gamma matrix $\gamma^{\mathrm{d}+1}$ has the following construction in terms of the other gamma matrices in d dimensions

$$
\gamma^{\mathrm{d}+1}=\frac{i^{\frac{\mathrm{d}-2}{2}}}{\mathrm{~d} !} \epsilon_{a_{1} \ldots a_{\mathrm{d}}} \gamma^{a_{1}} \ldots \gamma^{a_{\mathrm{d}}}
$$

where $a_{i}=0, \ldots, \mathrm{d}-1$ and $\epsilon$ is the totally antisymmetric Levi-Civita tensor on $\mathcal{M}$. Specifically, for $\mathrm{d}=4$, the gamma matrices for $G_{\mu \nu}$ are

$$
\begin{aligned}
& \tilde{\gamma}^{m}=\gamma^{m} \quad \text { when } m=0,1,2,3, \\
& \tilde{\gamma}^{\lambda}=-\frac{\lambda}{\lambda_{0}}\left(i \gamma^{5}+\lambda_{0} g_{m} \gamma^{m}\right) .
\end{aligned}
$$

The fifth gamma matrix $\gamma^{5}$ is crucial in discussions about chirality, which we will see when we apply the TW connection to spinor fields. Equation (75) shows that the volume bundle metric $G_{\mu \nu}$ explicitly builds in $\gamma^{5}$. Thus, we will expect our dynamical theory for $\mathcal{D}_{m n}$ to be chiral in nature when interacting with fermions. 
Equations (73), (74), (75) also serve to further establish the relationship between the projective gauge field $\mathcal{D}_{m n}$ and the notion of volume on $\mathcal{M}$. Any Lagrangian for $\mathcal{D}_{m n}$ will involve the metric $G_{\mu \nu}$ on $\mathcal{N}$, which in turn can be constructed from gamma matrices. Equation (73) says that one of these gamma matrices includes a rescaling of $\gamma^{\mathrm{d}+1}$ by $\lambda$, where $\gamma^{\mathrm{d}+1}$ is itself related to volume due to the presence of the epsilon tensor $\epsilon_{a_{1} \ldots a_{\mathrm{d}}}$. The epsilon tensor is alternating in its indices and transforms as a tensor density that is used to construct volume forms on $\mathcal{M}$. Therefore, we can again view $\lambda$ as a parameter which determines a rescaling of the volume element on $\mathcal{M}$.

\section{THE VIRASORO ALGEBRA AND PROJECTIVE GEOMETRY}

Here we will review three ways in which there is a correspondence between the projective connection's reduction to one dimension and the coadjoint elements of the Virasoro algebra. The Virasoro algebra $[14,39,40]$ may be regarded as the centrally extended algebra of vector fields in one dimension. Let $(\xi, a)$ and $(\eta, b)$ denote centrally extended vector fields in one dimension where $a$ and $b$ are elements in the center. Then the Lie algebra of these centrally extended vector fields is given through the commutator

$$
[(\xi, a),(\eta, b)]=\left(\xi \circ \eta,((\xi, \eta))_{0}\right),
$$

where $\xi \circ \eta$ is defined via

$$
\xi \circ \eta \equiv \xi^{a} \partial_{a} \eta^{b}-\eta^{a} \partial_{a} \xi^{b} .
$$

Here we explicitly expose the valence of the onedimensional vectors. The symbol $((\xi, \eta))_{0}$ is called the Gelfand-Fuchs two-cocycle [41] and is defined explicitly as

$$
\begin{aligned}
((\xi, \eta))_{0} & \equiv \frac{c}{2 \pi} \int\left(\xi \eta^{\prime \prime \prime}\right) d \theta \\
& =\frac{c}{2 \pi} \int \xi^{a} \nabla_{a}\left(g^{b c} \nabla_{b} \nabla_{c} \eta^{m}\right) g_{m n} d \theta^{n},
\end{aligned}
$$

where $g_{a b}$ is a one-dimensional metric. Equations (78) and (79) demonstrate an invariant pairing between $\xi$ and $\eta^{\prime \prime \prime}$. The Gelfand-Fuchs two-cocycle is an example of an invariant pairing between a vector and a quadratic differential $B$

$$
\begin{aligned}
\langle(\xi, a) \mid(B, c)\rangle & \equiv \int(\xi B) d \theta+a c \\
& =\int\left(\xi^{i} B_{i j}\right) d \theta^{j}+a c .
\end{aligned}
$$

In the Gelfand-Fuchs two-cocycle, the pairing is between a vector $\xi$ and a one-cocycle of $\eta$, where this one-cocycle is a projective transformation $[14,15]$ that has mapped the vector field $\eta$ into a quadratic differential. Explicitly,

$$
\eta \partial_{\theta} \rightarrow \eta^{\prime \prime \prime} d \theta^{2}=\nabla_{a}\left(g^{b c} \nabla_{b} \nabla_{c} \eta^{m}\right) g_{m n} d \theta^{a} d \theta^{n} .
$$

The invariant pairing in Eq. (80) follows if the action of another centrally extended algebra element, say $(\eta, d)$, leaves the pairing invariant, i.e.,

$$
(\eta, d) *\langle(\xi, a) \mid(B, c)\rangle=0 .
$$

This defines the coadjoint representation of the Virasoro algebra $[15,40]$.

$$
a d_{(\eta, d)}^{*}(B, c)=\left(\eta B^{\prime}+2 \eta^{\prime} B-c \eta^{\prime \prime \prime}, 0\right) .
$$

Then, a more general invariant two-cocycle relative to the centrally extended coadjoint element $\mathcal{B}=(B, c)$ can be written as

$$
(\xi, \eta)_{(B, c)}=\frac{c}{2 \pi} \int\left(\xi \eta^{\prime \prime \prime}-\xi^{\prime \prime \prime} \eta\right) d x+\frac{1}{2 \pi} \int\left(\xi \eta^{\prime}-\xi^{\prime} \eta\right) B d x
$$

One sees that the Gelfand-Fuchs case lives in the pure gauge sector, i.e., $\mathcal{B}=(0, c)$, of the space of coadjoint elements. It was also observed [15] that this action is the same as the action of the space of Sturm-Liouville operators on vector fields. Thus there is a correspondence

$$
(B, c) \Leftrightarrow-2 c \frac{d^{2}}{d x^{2}}+B(x),
$$

where on the left side $(B, c)$ is identified with a centrally extended coadjoint element of the Virasoro algebra and on the right side is a Sturm-Liouville operator with weight $c$ and $B(x)$ as the Sturm-Liouville potential.

\section{A. Correspondence through the transformation laws}

Here, we show how the relation between a coadjoint element of the Virasoro algebra and the Sturm-Liouville operator is reconciled by Thomas-Whitehead projective connections. We will evaluate the connection in one dimension where one can construct a Laplacian even though curvature is unavailable.

Consider the transformation of the diffeomorphism field $\mathcal{D}_{a b}$ in one dimension. One can show that in one dimension, Eq. (35), i.e.,

$$
\begin{aligned}
\mathcal{D}_{a b}^{\prime}= & \frac{\partial x^{m}}{\partial x^{\prime a}} \frac{\partial x^{n}}{\partial x_{b}^{\prime}} \mathcal{D}_{m n}-\frac{1}{(\mathrm{~d}+1)^{2}} \frac{\partial \log J}{\partial x^{\prime a}} \frac{\partial \log J}{\partial x^{\prime b}} \\
& -\frac{1}{\mathrm{~d}+1} \frac{\partial^{2} \log J}{\partial x^{\prime a} \partial x^{\prime b}}+\frac{1}{\mathrm{~d}+1} \frac{\partial \log J}{\partial x^{\prime c}} \Pi^{\prime c}{ }_{a b},
\end{aligned}
$$


reduces to [38]

$$
\delta \mathcal{D}=2 \xi^{\prime} \mathcal{D}+\mathcal{D}^{\prime} \xi-\frac{1}{2} \xi^{\prime \prime \prime}
$$

under an infinitesimal coordinate transformation. We may let $\mathcal{D}=q D$ where $q$ is an arbitrary constant. Then

$$
\begin{aligned}
\delta(q D) & =\delta \mathcal{D} \\
& =2 \xi^{\prime} \mathcal{D}+\mathcal{D}^{\prime} \xi-\frac{1}{2} \xi^{\prime \prime \prime} \\
& =2 \xi^{\prime} q D+q D^{\prime} \xi-\frac{1}{2} \xi^{\prime \prime \prime} \\
& =q\left(2 \xi^{\prime} D+D^{\prime} \xi-\frac{1}{2 q} \xi^{\prime \prime \prime}\right)
\end{aligned}
$$

or equivalently

$$
\delta D=2 \xi^{\prime} D+D^{\prime} \xi-\frac{1}{2 q} \xi^{\prime \prime \prime} .
$$

Choosing $q=\frac{1}{2 c}$, we see a correspondence between the one-dimensional Thomas projective connection and the coadjoint element in Eq. (83). This improves the argument made in [20].

\section{B. Correspondence through two-cocycles}

The covariant metric allows us to improve upon another correspondence between the projective connection and coadjoint elements discussed in [20]. We consider a projective two-cocycle on $\mathcal{N}$ for a path $C$ as

$$
\begin{aligned}
\langle\xi, \eta\rangle_{(\zeta)}= & q \int_{C(\zeta)} \xi^{\alpha}\left(\tilde{\nabla}_{\alpha} G^{\rho \nu} \tilde{\nabla}_{\rho} \tilde{\nabla}_{\nu} \eta^{\beta} G_{\beta \mu}\right) \zeta^{\mu} d \sigma \\
& -(\xi \leftrightarrow \eta),
\end{aligned}
$$

where $\sigma$ parametrizes the path. The vector $\zeta^{\mu} \equiv \frac{d x^{\mu}}{d \sigma}$ defines the path $C$. Here, the coordinates on $\mathcal{N}$ are $x^{\alpha}=(x, \lambda)$. We choose the vector fields as $\xi^{\beta}=\left(\xi^{b},-\lambda \xi^{a} g_{a}\right)$ and $\eta^{\beta}=\left(\eta^{b},-\lambda \eta^{a} g_{a}\right)$. Consider a path given by a fixed value $\lambda=\lambda_{0}$ along the vector $\zeta_{\lambda_{0}}^{\mu}=\left(\frac{d x}{d \sigma}, 0\right)$. The metric used to construct the projective Laplacian is the one-dimensional version of Eq. (64). Setting the metric to a constant $g_{11}$ and the components of the vector fields to $\xi_{1}$ and $\eta_{1}$, respectively and keeping in mind that $\Pi_{b c}^{a}=0$ in one dimension, one finds that

$$
\begin{aligned}
\langle\xi, \eta\rangle_{\left(\zeta_{\lambda_{0}}\right)}= & q \int \xi_{1}\left(2 \mathcal{D}_{11}-g_{11} \frac{1}{\lambda_{0}^{2}}\right) \eta_{1}^{\prime} d x \\
& +q \int \xi_{1} \eta_{1}^{\prime \prime \prime} d x-(\xi \leftrightarrow \eta) .
\end{aligned}
$$

Comparing this to Eq. (84), we make the observation that the projective connection and the coadjoint element $(B, q)$ are in correspondence through

$$
2 q \mathcal{D}_{11}-\frac{q}{\lambda_{0}^{2}}=B
$$

which recovers Eq. (84) for $q=\frac{c}{2 \pi}$.

\section{Correspondence through gauge invariant action}

Using the action in [20], we write the invariant projective Einstein-Hilbert terms as

$$
\begin{aligned}
S_{\mathrm{PEH}} & =\int d^{2} x d \lambda \sqrt{|G|} \mathcal{K}_{\alpha \beta} G^{\alpha \beta}=\int d^{2} x d \lambda \sqrt{|G|} \mathcal{K} \\
& =\lambda_{0}\left[\int d \lambda \frac{1}{\lambda}\right] \int d^{2} x \sqrt{|g|}\left(R+g^{a b}\left(2 \mathcal{P}_{b a}-\mathcal{P}_{a b}\right)\right) \\
& =\beta\left(S_{\mathrm{EH}}+\int d^{2} x \sqrt{|g|} g^{a b}\left(2 \mathcal{P}_{b a}-\mathcal{P}_{a b}\right)\right)
\end{aligned}
$$

where we have used the projective Schouten tensor to write this in terms of the Riemann scalar curvature for familiarity. In two dimensions, the Einstein-Hilbert term is the GaussBonnet topological invariant. The Polyakov metric has constant volume and $\mathcal{D}_{a b}$ and $\mathcal{P}_{a b}$ are equivalent. Evaluating this on the Polyakov metric in two dimensions gives the coupling to the coadjoint element

$$
S_{\text {Polyakov Coupling }}=\int d^{2} \theta \mathcal{P}_{++} h_{--} .
$$

Again, the importance of this is to show dimensional universality of the interaction term in the Polyakov action as $\sqrt{|G|} \mathcal{K}$ has meaning in any dimension. Thus, $\mathcal{D}_{a b}$ is to the Virasoro algebra of one-dimensional centrally extended vector fields as the Yang-Mills gauge field $A_{a}$ is to affine Lie algebras in one dimension. Furthermore, the projective curvature $\mathcal{K}^{\alpha}{ }_{\mu \nu \beta}$ can be used to build dynamical theories for $\mathcal{D}_{a b}$ just as the gauge curvature $F_{a b}$ can provide dynamics for the gauge fields related to external gauge symmetries.

\section{SPINOR FIELDS ON $\mathcal{N}$}

To this point, we have discussed the representation theory for the Thomas-Whitehead connection as related to tensors. Now we examine the relation among projective connections, spinors, and their associated Dirac equation. We will focus on spin $\frac{1}{2}$ spinors throughout.

\section{A. The spin covariant derivative}

To construct the spin connection for the generalized metric $G_{\alpha \beta}$ we will need the frame fields that make contact with the Minkowski space metric on the Thomas cone. There are several types of indices involved. First, there is a 
distinction between spacetime indices and the extra $\lambda$ coordinate on $\mathcal{N}$. Second, there is a distinction between curved indices and flat indices. To make calculations clear, we will adopt the following conventions for indices:

(i) Greek indices $\mu, \nu$ : curved coordinates on $\mathcal{N}$,

(ii) Latin indices $m, n$ : curved coordinates on $\mathcal{M}$,

(iii) underlined indices $\underline{a}, \underline{\alpha}$ : flat coordinates on $\mathcal{M}, \mathcal{N}$,

(iv) $\lambda$ : volume bundle coordinate,

(v) 5: flattened volume bundle coordinate.

We use the number $\underline{5}$ to represent the extra coordinate in flat space, due to the case of four-dimensional spacetime, where the gamma matrices are commonly labeled $5,3,2,1,0$ for historical reasons. For the metric $g_{m n}$ on $\mathcal{M}$, $e^{m}{ }_{\underline{a}}$ (with inverse $e^{\underline{a}}{ }_{m}$ ) are the associated frame fields satisfying

$$
\begin{aligned}
g_{m n} & =e^{\underline{a}}{ }_{m} e^{\underline{b}}{ }_{n} \eta_{\underline{a b}}, \\
\eta_{\underline{a b}} & =e^{m}{ }_{\underline{a}} e^{n}{ }_{\underline{b}} g_{m n} .
\end{aligned}
$$

Similarly, the frame fields denoted $\tilde{e}^{\mu}{ }_{\underline{\alpha}}$ will be associated with the metric $G_{\mu \nu}$ on $\mathcal{N}$, and the indices range over all dimensions, including $\lambda$ for the curved coordinates on $\mathcal{N}$

$$
\begin{aligned}
& G_{\alpha \beta}=\tilde{e}^{\mu}{ }_{\alpha}^{\mu} \tilde{e}^{\underline{\nu}}{ }_{\beta} \tilde{\eta}_{\mu \underline{\nu}}, \\
& \tilde{\eta}_{\underline{\alpha} \underline{\beta}}=\tilde{e}^{\mu}{ }_{\alpha} \tilde{e}^{\nu}{ }_{\underline{\beta}} G_{\mu \nu} .
\end{aligned}
$$

We may also use the frame fields to write the components of the Dirac matrices in curved spacetime coordinates

$$
\tilde{\gamma}^{\mu}=\tilde{e}^{\mu}{ }_{\underline{\gamma}} \underline{\gamma}^{\underline{\alpha}}
$$

For the metric $G_{\mu \nu}$ given by Eq. (60), the frame fields are listed as follows:

$$
\begin{aligned}
\tilde{e}^{m}{ }_{\underline{a}} & =e^{m}{ }_{\underline{a}}, \\
\tilde{e}^{m}{ }_{\underline{5}} & =0, \\
\tilde{e}^{\lambda} & =-\lambda e^{m}{ }_{\underline{a}} g_{m}, \\
\tilde{e}^{\lambda}{ }_{\underline{5}} & =\frac{\lambda}{\lambda_{0}} .
\end{aligned}
$$

The inverse frame field components are then given by:

$$
\begin{aligned}
\tilde{e}^{\underline{a}}{ }_{m} & =e^{\underline{a}}{ }_{m}, \\
\tilde{e}^{\underline{5}} & =\lambda_{0} g_{m}, \\
\tilde{e}^{\underline{a}}{ }_{\lambda} & =0, \\
\tilde{e}^{\underline{5}} & =\frac{\lambda_{0}}{\lambda} .
\end{aligned}
$$

Let $\tilde{\Gamma}^{\mu}{ }_{\nu \rho}$ be the components of the TW connection, and call $\stackrel{\circ}{\nabla}_{\mu}$ the corresponding covariant derivative operator that acts only on the curved indices as opposed to flat indices. Define

$$
\tilde{\omega}^{\mu}{ }_{\underline{\alpha}}=\stackrel{\circ}{\nabla}_{\nu} \tilde{e}_{\underline{\alpha}}^{\mu}=\partial_{\nu} \tilde{e}_{\underline{\alpha}}^{\mu}+\tilde{\Gamma}_{\rho \nu}^{\mu} \tilde{e}^{\rho}{ }_{\underline{\alpha}} .
$$

We use the geometric object $\tilde{\omega}$ of Eq. (99) to define a new spin covariant derivative

$$
\begin{aligned}
& \tilde{D}_{\mu} V^{\underline{\alpha}}=\partial_{\mu} V^{\underline{\alpha}}+\tilde{\omega}^{\underline{\alpha}} \underline{\underline{\beta} \mu} V^{\underline{\beta}}, \\
& \tilde{D}_{\mu} V_{\underline{\alpha}}=\partial_{\mu} V_{\underline{\alpha}}-\tilde{\omega}^{\underline{\beta}} \underline{\alpha}^{\alpha} V_{\underline{\beta}},
\end{aligned}
$$

which recognizes tensorial objects, such as the vector $V^{\underline{\alpha}}$, written in flat coordinates. We can take the full covariant derivative of a geometric object with curved and flat spacetime indices by using the ordinary connection coefficients $\tilde{\Gamma}_{\nu \rho}^{\mu}$ for curved indices and the spin connection coefficients $\tilde{\omega}_{\underline{\alpha} \nu}^{\mu}$ for flat indices. From now on, we will denote this full covariant derivative operator by $\tilde{\nabla}_{\mu}$. By construction, the frame fields are covariantly constant, satisfying

$$
\tilde{\nabla}_{\nu} \tilde{e}_{\underline{\alpha}}^{\mu}=0=\tilde{\nabla}_{\nu} \tilde{e}^{\underline{\alpha}}{ }_{\mu}
$$

Then for any vector $V^{\mu}$, we have

$$
\tilde{\nabla}_{\nu} V^{\underline{\alpha}}=\tilde{e}^{\underline{\alpha}}{ }_{\mu} \tilde{\nabla}_{\nu} V^{\mu}
$$

so that the frame fields can be used to change indices without having to introduce an extra derivative term.

With the frame fields on hand, we can calculate the coefficients of the TW spin connection using Eq. (99). Recall the TW connection coefficients originally presented in Eq. (22):

$$
\begin{aligned}
\tilde{\Gamma}_{b c}^{a} & =\Pi_{b c}^{a}, \\
\tilde{\Gamma}_{b c}^{\lambda} & =\lambda \mathcal{D}_{b c}, \\
\tilde{\Gamma}_{\lambda b}^{a} & =\tilde{\Gamma}_{b \lambda}^{a}=\frac{1}{\lambda} \delta^{a}{ }_{b} .
\end{aligned}
$$

We simply need to plug these coefficients and the frame fields into Eq. (99) to get the TW spin coefficients that we desire. For example, if $\underline{a}, \underline{b} \neq \underline{5}$ and $\mu \neq \lambda$ (which aligns with our chosen index conventions), we have

$$
\begin{aligned}
& \tilde{\omega}_{\underline{a b m}}=\left(\partial_{m} \tilde{e}_{\underline{b}}^{n}+\tilde{\Gamma}_{\rho m}^{n} \tilde{e}_{\underline{\rho}}^{\rho}\right) \tilde{e}^{-}{ }_{n} \eta_{\underline{a c}} \\
& +\left(\partial_{m} \tilde{e}_{\underline{b}}^{\lambda}+\tilde{\Gamma}_{\rho m}^{\lambda} \tilde{e}^{\rho}{ }_{b}\right) \tilde{e}^{\underline{c}}{ }_{\lambda} \eta_{\underline{a c}} \\
& +\left(\partial_{m} \tilde{e}^{n}{ }_{b}+\tilde{\Gamma}_{\rho m}^{n} \tilde{e}^{\rho}{ }_{b}\right) \tilde{e}^{-}{ }_{n} \eta_{a \underline{5}} \\
& +\left(\partial_{m} \tilde{e}_{\underline{b}}^{\lambda}+\tilde{\Gamma}_{r m}^{\lambda} \tilde{e}^{r}{ }_{b}\right) \tilde{e}^{\underline{5}}{ }_{\lambda} \eta_{\underline{a}},
\end{aligned}
$$

and since $\eta_{\underline{a} \underline{5}}=0$ and $\tilde{e}^{\underline{c}} \lambda=0$, this reduces to 


$$
\begin{aligned}
\tilde{\omega}_{\underline{\omega} b m} & =\left(\partial_{m} \tilde{e}^{n}{ }_{\underline{b}}+\tilde{\Gamma}_{\rho m}^{n}{ }_{\rho m} \tilde{e}^{\rho}{ }_{\underline{b}}\right) \tilde{e}^{\underline{c}}{ }_{n} \eta_{\underline{a c}} \\
& =\left(\partial_{m} e^{n}{ }_{\underline{b}}+\Gamma_{r m}^{n}{ }_{r m} e_{\underline{b}}+\delta^{n}{ }_{r} \alpha_{m} e^{r}{ }_{\underline{b}}-\delta^{n}{ }_{m} \Delta_{r} e^{r} \underline{b}\right) e^{\underline{c}}{ }_{n} \eta_{\underline{a c}} \\
& =\omega_{\underline{a b m}}+\alpha_{m} \eta_{\underline{a b}}+e^{r}{ }_{\underline{b}} e^{\underline{c}}{ }_{m} \eta_{\underline{a c}} \Delta_{r},
\end{aligned}
$$

where $\omega_{\underline{a} \underline{b} m}$ (without a tilde) is the coefficient of the spin connection for the underlying spacetime connection $\Gamma_{p m}^{n}$. Thus, we see how the TW spin connection coefficients $\tilde{\omega}_{\underline{a} \underline{b} m}$ are offset from the spacetime spin connection coefficients $\omega_{\underline{a} \underline{b} m}$ for $\underline{a}, \underline{b} \neq \underline{5}$. Below, we present the full list of independent TW spin connection coefficients:

$$
\begin{aligned}
& \tilde{\omega}_{\underline{a b m}}=\omega_{\underline{a b m}}+\alpha_{m} \eta_{\underline{a b}}-e_{\underline{b}}^{r} e^{\underline{c}}{ }_{m} \eta_{\underline{a c}} \Delta_{r}, \\
& \tilde{\omega}_{\underline{a b \lambda}}=\frac{1}{\lambda} \eta_{\underline{a b}}, \\
& \tilde{\omega}_{\underline{a 5} m}=\eta_{\underline{a b}} e^{\underline{c}}{ }_{m} \frac{1}{\lambda_{0}}, \\
& \tilde{\omega}_{\underline{5} \underline{b} m}=-\lambda_{0} e_{\underline{b}}^{p}\left(\mathcal{D}_{p m}-\partial_{m} g_{p}+\Gamma_{p m}^{n} g_{n}+\alpha_{m} g_{m}-\Delta_{m} g_{p}\right) \text {, } \\
& \tilde{\omega}_{\underline{a} \underline{ } \lambda}=\tilde{\omega}_{\underline{5} \underline{b} \lambda}=\tilde{\omega}_{\underline{5} \underline{5} \mathrm{~m}}=0 \text {, } \\
& \tilde{\omega}_{\underline{5} \underline{5} \lambda}=-\frac{1}{\lambda} \text {. }
\end{aligned}
$$

In Eqs. (105) and (106), we have explicitly written out $\Pi_{\rho \mu}^{\nu}$ in terms of a member of the equivalence class, $\Gamma^{a}{ }_{b c}$ and its trace $\alpha_{a}$. This allows us to see the relationship to the spin connection on $\mathcal{M}$. It is clear that $\tilde{\omega}_{\underline{a} \underline{b} \rho}$ is not antisymmetric in $\underline{a}$ and $\underline{b}$.

\section{B. The spinor connection}

Let $\Psi(x)$ and $\bar{\Psi}(x)$ be a spinor field and its Pauli adjoint, respectively representing a fermion and its antipartner on the manifold $\mathcal{N}$. Then the covariant derivative acting on the spinor is

$$
\tilde{\nabla}_{\mu}=\partial_{\mu}+\tilde{\Omega}_{\mu},
$$

where

$$
\tilde{\Omega}_{\mu}=\frac{1}{4} \tilde{\omega}_{\underline{\alpha} \underline{\beta}} \tilde{\gamma}^{\underline{\alpha}} \tilde{\gamma}^{\beta},
$$

and

$$
\tilde{\nabla}_{\mu} \Psi=\partial_{\mu} \Psi+\tilde{\Omega}_{\mu} \Psi=\partial_{\mu} \Psi+\frac{1}{4} \tilde{\omega}_{\underline{\alpha}} \underline{\mu} \tilde{\gamma}^{\alpha} \tilde{\gamma}^{\beta} \Psi .
$$

Similarly,

$$
\nabla_{\mu} \bar{\Psi}=\partial_{\mu} \bar{\Psi}-\bar{\Psi} \tilde{\Omega}_{\mu}=\partial_{\mu} \bar{\Psi}-\frac{1}{4} \tilde{\omega}_{\underline{\alpha}} \underline{\mu} \mu \bar{\Psi} \tilde{\gamma}^{\alpha} \tilde{\gamma}^{\underline{\beta}} .
$$

The spin connection in Eqs. (108) and (109) have in general both symmetric and antisymmetric components in their flat indices $a, b$. This is because the connection $\tilde{\Gamma}^{\mu}{ }_{\nu \rho}$ on $\mathcal{N}$ is not a metric compatible connection, since $\tilde{\nabla}$ cannot be made metric compatible. The enveloping algebra of the gamma matrices is thus

$$
\tilde{\gamma}^{\underline{\alpha}} \tilde{\gamma}^{\underline{\beta}}=-i \sigma^{\underline{\alpha}} \underline{\beta}+\tilde{\eta}^{\underline{\alpha}}-\underline{\beta} I_{4},
$$

where the Sigma matrices generate the local $S O(4,1)$ Lorentz algebra on the Thomas cone, i.e.,

$\left[\sigma^{\underline{\alpha} \underline{\beta}}, \sigma^{\underline{\mu}} \underline{\underline{\nu}}\right]=-2 i\left(\eta^{\underline{\alpha} \underline{\mu}} \sigma^{\underline{\beta}} \underline{\underline{\nu}}+\eta^{-\underline{\beta}}-\sigma^{-\underline{\alpha}} \underline{\mu}-\eta^{\underline{\alpha}}-\sigma^{\underline{\beta}}-\underline{\mu}-\eta^{\underline{\beta}}-\underline{\mu} \sigma^{\underline{\alpha}}-\right)$.

The $\tilde{\omega}_{[\underline{\alpha} \underline{\beta}] \mu}$ therefore correspond to gauge fields for the local Lorentz transformation, while the $\tilde{\omega}_{(\underline{\alpha} \underline{\beta}) \mu}$ generate a translation on the fermions to their tensor densities. Let us write $\Omega_{\mu}=\Omega_{\mu}^{S}+\Omega_{\mu}^{A}$, such that the symmetric component is

$$
\tilde{\Omega}_{\rho}^{S}=\tilde{\omega}_{(\underline{\alpha} \underline{\beta}) \rho} \tilde{\eta}^{\underline{\alpha}} \underline{\beta}=(\mathrm{d}+1) \tilde{\omega}_{\rho}
$$

and the expected $\mathrm{SO}(4,1)$ connection is

$$
\tilde{\Omega}_{\rho}^{A}=-i \tilde{\omega}_{[\underline{\alpha} \underline{\beta}]} \tilde{\sigma}^{\underline{\alpha} \underline{\beta}} .
$$

In Eq. (113), the space-time component of this Abelian connection is $\Gamma_{a c}^{a} \equiv \Gamma_{c}$. In differential geometry, such a term appears in the presence of weighted spinors [42] that transform relative to an unweighted spinor $\phi$ as

$$
\phi_{v w}=\operatorname{det}(g) \frac{\left(v I_{4}+w_{\gamma}\right)^{2}}{2} \phi
$$

in four dimensions. The spinor $\psi_{v w}$ is said to have weight $\left(v I_{4}+w \gamma^{5}\right)$. For these weighted spinors, the spin connection is augmented to be [42]

$$
\Omega_{m} \rightarrow \Omega_{m}+\left(v I_{4}+w \gamma^{5}\right) \Gamma_{m} .
$$

We use this to define spinor representations ( $\frac{1}{2}$ integer spin) on the Thomas cone. First, we remark that on the Thomas cone $\gamma^{5}$ is an invariant tensor since

$$
\lambda_{0} \gamma^{5}=\Upsilon{ }^{\underline{\alpha_{1}}} \epsilon_{\underline{\alpha}_{1} \cdots \underline{\alpha}_{5}} \gamma^{\underline{\alpha}_{2} \cdots \gamma^{\alpha_{5}},}
$$

where $\Upsilon^{\underline{\nu}}=\tilde{e}^{\underline{\underline{ }}}{ }_{\mu} \Upsilon^{\mu}$. Since $\lambda \rightarrow \lambda|J|^{\frac{-1}{\mathrm{~d}+1}}$, we can expect weighted spinor representations on the Thomas cone to be

$$
\Psi\left(x^{a}, \lambda\right)=\left(\frac{\lambda}{\lambda_{0}}\right)^{\frac{(\mathrm{d}+1)}{2}\left(v I_{4}+w \gamma^{5}\right)} \phi\left(x^{a}\right)
$$

and 


$$
\bar{\Psi}\left(x^{a}, \lambda\right)=\bar{\phi}\left(x^{a}\right)\left(\frac{\lambda}{\lambda_{0}}\right)^{-\frac{(\mathrm{d}+1)}{2}\left(v I_{4}+w \gamma^{5}\right)} .
$$

Note that

$$
\left(\frac{\lambda}{\lambda_{0}}\right)^{M}=e^{\left(\log \left(\frac{\lambda}{\lambda_{0}}\right)\right) M}
$$

for a matrix $M$ and

$$
\lambda \frac{\partial}{\partial_{\lambda}}\left(\frac{\lambda}{\lambda_{0}}\right)^{M}=M\left(\frac{\lambda}{\lambda_{0}}\right)^{M}
$$

\section{The gauge invariant TW Dirac action}

Equation (109) is the general expression for the covariant derivative of a spinor field. For the TW connection, we will use the spin connection coefficients given by Eq. (106) and decompose the connection into its chiral and nonchiral parts. This will illuminate the nature of the TW spinor connection on $\mathcal{M}$. With this, $\not \nabla$ becomes

$$
\begin{aligned}
\tilde{\nabla} \Psi= & \tilde{\gamma}^{\alpha} \tilde{\nabla}_{\alpha} \Psi=\tilde{\gamma}^{m} \partial_{m} \Psi+\tilde{\gamma}^{\lambda} \partial_{\lambda} \Psi \\
& +\frac{1}{4}\left(\tilde{\gamma}^{m} \tilde{\omega}_{\underline{a}} \underline{b} m \tilde{\gamma}^{\underline{a}} \tilde{\gamma}^{\underline{b}}+\tilde{\gamma}^{\lambda} \tilde{\omega}_{\underline{a}} \underline{b} \lambda \tilde{\gamma}^{a} \tilde{\gamma}^{\underline{b}}\right. \\
& \left.+\tilde{\gamma}^{m} \tilde{\omega}_{5 \underline{b} m} \tilde{\gamma}^{5} \tilde{\gamma}^{\underline{b}}+\tilde{\gamma}^{\lambda} \tilde{\omega}_{55 \lambda} \tilde{\gamma}^{5} \tilde{\gamma}^{5}\right) \Psi .
\end{aligned}
$$

And for $\bar{\Psi}$,

$$
\begin{aligned}
\tilde{\nabla} \bar{\Psi}= & \left(\tilde{\nabla}_{\alpha} \bar{\Psi}\right) \tilde{\gamma}^{\alpha}=\partial_{m} \bar{\Psi} \tilde{\gamma}^{m}+\partial_{\lambda} \bar{\Psi} \tilde{\gamma}^{\lambda} \\
& -\frac{1}{4} \bar{\Psi}\left(\tilde{\omega}_{\underline{a}} \underline{b} m \tilde{\gamma}^{\underline{a}} \tilde{\gamma}^{\underline{b}} \tilde{\gamma}^{m}+\tilde{\omega}_{\underline{a}} \underline{b} \lambda \tilde{\gamma}^{\underline{a}} \tilde{\gamma}^{\underline{b}} \tilde{\gamma}^{\lambda}\right. \\
& \left.+\tilde{\omega}_{5 \underline{b} m} \tilde{\gamma}^{5} \tilde{\gamma}^{\underline{b}} \tilde{\gamma}^{m}+\tilde{\omega}_{55 \lambda} \tilde{\gamma}^{5} \tilde{\gamma}^{5} \tilde{\gamma}^{\lambda}\right) .
\end{aligned}
$$

Evaluating these with the coefficients from Eq. (106) yields

$$
\begin{aligned}
\tilde{\nabla} \Psi= & \not{\nabla} \Psi-i \frac{\lambda}{\lambda_{0}} \gamma^{5}\left(\partial_{\lambda} \Psi\right)-\lambda g_{m} \gamma^{m}\left(\partial_{\lambda} \Psi\right) \\
& +\frac{1}{4}\left[\mathrm{~d}\left(\alpha_{m}-g_{m}\right)+\mathrm{d} \alpha_{m}-g_{m}\right] \gamma^{m} \Psi \\
& -\frac{1}{4} i\left[\lambda_{0}\left(\mathcal{D}_{r m}-\partial_{m} g_{r}+\Gamma^{n}{ }_{r m} g_{n}+\alpha_{m} g_{r}-\Delta_{m} g_{r}\right) g^{m r}\right. \\
& \left.+\frac{1}{\lambda_{0}}(\mathrm{~d}+1)\right] \gamma^{5} \Psi
\end{aligned}
$$

while

$$
\begin{aligned}
\tilde{\nabla} \bar{\Psi}= & \not \bar{\Psi}+i \frac{\lambda}{\lambda_{0}}\left(\partial_{\lambda} \bar{\Psi}\right) \gamma^{5}+\lambda g_{m}\left(\partial_{\lambda} \bar{\Psi}\right) \gamma^{m} \\
& -\frac{1}{4}\left[\mathrm{~d}\left(\alpha_{m}-g_{m}\right)+\mathrm{d} \alpha_{m}-g_{m}\right] \bar{\Psi} \gamma^{m} \\
& +\frac{1}{4} i\left[\lambda_{0}\left(\mathcal{D}_{r m}-\partial_{m} g_{r}+\Gamma_{r m}^{n}{ }_{r m} g_{n}+\alpha_{m} g_{r}-\Delta_{m} g_{r}\right) g^{m r}\right. \\
& \left.+\frac{1}{\lambda_{0}}(\mathrm{~d}+1)\right] \bar{\Psi} \gamma^{5} .
\end{aligned}
$$

Here, $\nabla_{m}$ (without a tilde) is the spinor covariant derivative operator associated with the space-time connection $\Gamma^{m}{ }_{n r}$. Using this decomposition, we write Eq. (122) as

$$
\begin{aligned}
\tilde{\nabla} \Psi= & \not \nabla \Psi-i \frac{\lambda}{\lambda_{0}} \gamma^{5}\left(\partial_{\lambda} \Psi\right)-\lambda g_{m} \gamma^{m}\left(\partial_{\lambda} \Psi\right) \\
& +B_{m} \gamma^{m} \Psi-i \frac{1}{4} \Xi \gamma^{5} \Psi
\end{aligned}
$$

where we have defined $B_{m}$ and $\Xi$ as

$$
\begin{gathered}
B_{m} \equiv \frac{1}{4}\left(\mathrm{~d}\left(\alpha_{m}-g_{m}\right)+\mathrm{d} \alpha_{m}-g_{m}\right), \\
\Xi \equiv \lambda_{0}\left(\mathcal{D}_{r m}-\partial_{m} g_{r}+\Gamma^{n}{ }_{r m} g_{n}+\alpha_{m} g_{r}-\Delta_{m} g_{r}\right) g^{m r} \\
+\frac{1}{\lambda_{0}}(\mathrm{~d}+1) .
\end{gathered}
$$

The TW Dirac Lagrangian density that yields the Dirac equation

$$
i \tilde{\nabla} \Psi-\left(M+i M_{\chi} \gamma^{5}\right) \Psi=0
$$

for a mass $M$ and a chiral mass $M_{\chi}$, may be written explicitly in covariant and self-conjugate form as

$$
\begin{aligned}
\mathcal{L}_{\mathrm{TWD}}= & \frac{i}{2} \sqrt{|G|} G^{\mu \nu}\left(\bar{\Psi} \tilde{\gamma}_{\mu}\left(\partial_{\nu}+\tilde{\Omega}_{\nu}\right) \Psi-\left(\partial_{\nu} \bar{\Psi}-\bar{\Psi} \tilde{\Omega}_{\nu}\right) \tilde{\gamma}_{\mu} \Psi\right) \\
& -\sqrt{|G|}\left(M \bar{\Psi} \Psi+i M_{\chi} \bar{\Psi} \gamma^{5} \Psi\right) \\
& -\frac{i}{2} \bar{\Psi} \tilde{\nabla}_{\mu}\left(\sqrt{|G|} \tilde{\gamma}^{\mu}\right) \Psi
\end{aligned}
$$

The last term arises because the metric and covariant derivative operator are not compatible since

$$
\tilde{\nabla}_{\mu} \tilde{\gamma}^{\mu}=\partial_{\mu} \tilde{\gamma}^{\mu}+\tilde{\Gamma}_{\mu \alpha}^{\mu} \tilde{\gamma}^{\alpha}+\left[\tilde{\Omega}_{\alpha}, \tilde{\gamma}^{\alpha}\right]
$$

does not vanish. The commutator term is precisely where the field $\mathcal{D}_{m n}$ resides. We can rewrite this so that the field equations on $\Psi$ (or $\bar{\Psi}$ ) are explicit if we integrate by parts the derivative term on $\bar{\Psi}$. Then 


$$
\begin{aligned}
-\frac{i}{2} \sqrt{|G|} G^{\mu \nu}\left(\partial_{\nu} \bar{\Psi}\right) \tilde{\gamma}_{\mu} \Psi= & -\partial_{\nu}\left(\frac{i}{2} \sqrt{|G|} G^{\mu \nu} \bar{\Psi} \tilde{\gamma}_{\mu} \Psi\right) \\
& +\bar{\Psi} \partial_{\nu}\left(\frac{i}{2} \sqrt{|G|} G^{\mu \nu} \tilde{\gamma}_{\mu} \Psi\right) \\
= & -\underbrace{\partial_{\lambda}\left(\frac{i}{2} \sqrt{|G|} G^{\lambda \lambda} \bar{\Psi} \tilde{\gamma}_{\lambda} \Psi\right)}_{\text {total } \lambda \text { derivative }} \\
& -\underbrace{\partial_{a}\left(\frac{i}{2} \sqrt{|G|} G^{a b} \bar{\Psi} \tilde{\gamma}_{b} \Psi\right)}_{\text {total space-time derivative }} \\
& +\bar{\Psi} \partial_{\nu}\left(\frac{i}{2} \sqrt{|G|} \tilde{\gamma}^{\nu} \Psi\right) .
\end{aligned}
$$

The total space-time derivative may be eliminated on the boundary. However the total $\lambda$ derivative will in general be finite and could contribute to the field equations. Let us examine this term more carefully. One sees that

$$
\begin{gathered}
\partial_{\lambda}\left(\frac{i}{2} \sqrt{|G|} G^{\lambda \lambda} \bar{\Psi} \tilde{\gamma}_{\lambda} \Psi\right)=\partial_{\lambda}\left(\frac{i}{2} \sqrt{|G|} \bar{\Psi} \tilde{\gamma}^{\lambda} \Psi\right) \\
=-\partial_{\lambda}\left(\frac{i}{2} \frac{\lambda_{0}}{\lambda} \sqrt{|g|} \bar{\Psi} \frac{\lambda}{\lambda_{0}}\left(i \gamma^{5}+\lambda_{0} g_{m} \gamma^{m}\right) \Psi\right) \\
=\frac{i}{2} \sqrt{|g|} \partial_{\lambda}\left(i \bar{\Psi} \gamma^{5} \Psi+\lambda_{0} g_{m} \bar{\Psi} \gamma^{m} \Psi\right) .
\end{gathered}
$$

From the spinor projective representations in Eqs. (117) and (118), this will vanish when $w=0$, eliminating any chiral density terms. We also observe that the term $g_{m} \bar{\Psi} \gamma^{m} \Psi$ would vanish if the coordinates were gauge fixed so that $g_{m}=0$ (constant volume). Had we used a constant volume metric, this condition would have gone unnoticed. This also guarantees that the action is a scalar. The remaining term in Eq. (131) leads to the last summand in the covariant Lagrangian density, Eq. (128). With this, we write the Lagrangian which realizes the Dirac equation on $\Psi$ as

$$
\begin{aligned}
\mathcal{L}_{\mathrm{TWD}}= & \frac{\lambda_{0}}{\lambda} \sqrt{|g|}\left(i \bar{\Psi} \not \nabla \Psi+\frac{\lambda}{\lambda_{0}} \bar{\Psi} \gamma^{5} \partial_{\lambda} \Psi-i \lambda g_{m} \bar{\Psi} \gamma^{m} \partial_{\lambda} \Psi\right) \\
& +\frac{\lambda_{0}}{\lambda} \sqrt{|g|}\left(i B_{m} \bar{\Psi} \gamma^{m} \Psi+\Xi \bar{\Psi} \gamma^{5} \Psi\right) \\
& -\frac{\lambda_{0}}{\lambda} \sqrt{|g|}\left(M \bar{\Psi} \Psi+i M_{\chi} \bar{\Psi} \gamma^{5} \Psi\right) .
\end{aligned}
$$

Had we wished to add a Yang-Mills potential to the action, we would have a term

$$
\begin{aligned}
\mathcal{L}_{\mathrm{YM}} & =\sqrt{|G|} \bar{\Psi} \tilde{\gamma}^{\mu} \tilde{A}_{\mu} \Psi \\
& =\sqrt{|G|}\left(-i \frac{1}{\lambda_{0}} \bar{\Psi} \gamma^{5} \Psi+\bar{\Psi} \gamma^{a} A_{a} \Psi\right),
\end{aligned}
$$

where a chiral mass term $M_{\mathcal{A}}=\frac{1}{\lambda_{0}}$ is induced. This follows since the corresponding projective one-form for the matrix valued potential is

$$
\tilde{A}_{\mu}=\left(A_{a}+g_{a} \mathbf{1}, \frac{1}{\lambda} \mathbf{1}\right),
$$

where 1 is in the center of the algebra. Then, using Eq. (73), we have the result

$$
\tilde{\gamma}^{\mu} \tilde{A}_{\mu}=-i \frac{1}{\lambda_{0}} \gamma^{5} \mathbf{1}+\gamma^{a} A_{a}
$$

In the Lagrangian density, Eq. (135), we have left terms with explicit $\lambda$ dependence of $\Psi$. Following Eqs. (117) and (118), along with the requirement that the action be a scalar, we have

$$
\begin{aligned}
& \Psi(x, \lambda)=\left(\frac{\lambda}{\lambda_{0}}\right)^{\frac{v}{2}(\mathrm{~d}+1)} \phi\left(x^{a}\right), \\
& \bar{\Psi}(x, \lambda)=\left(\frac{\lambda}{\lambda_{0}}\right)^{-\frac{v}{2}(\mathrm{~d}+1)} \bar{\phi}(x),
\end{aligned}
$$

where $v$ is the density weight which determines precisely how $\Psi$ will transform under $\lambda \rightarrow \lambda^{\prime}$. In the TW Dirac Lagrangian $\mathcal{L}_{\text {TWD }}$ of Eq. (135), this representation of $\Psi$ will only affect the terms

$$
\begin{aligned}
& \frac{\lambda}{\lambda_{0}} \bar{\Psi} \gamma^{5}\left(\partial_{\lambda} \Psi\right)-i \lambda g_{m} \bar{\Psi} \gamma^{m}\left(\partial_{\lambda} \Psi\right) \\
& \quad=\frac{v(\mathrm{~d}+1)}{2 \lambda_{0}} \bar{\Psi} \gamma^{5} \Psi-i \frac{v}{2}(\mathrm{~d}+1) g_{m} \bar{\Psi} \gamma^{m} \Psi
\end{aligned}
$$

so that the TW Dirac Lagrangian can be reduced to a Lagrangian on $\psi$ with $v$ a weight parameter

$$
\begin{aligned}
\mathcal{L}_{\mathrm{TWD}}= & \frac{\lambda_{0}}{\lambda} \sqrt{|g|}(i \bar{\phi} \tilde{\dot{\phi}} \phi-M \bar{\phi} \phi) \\
= & \frac{\lambda_{0}}{\lambda} \sqrt{|g|}(i \bar{\phi} \not \boldsymbol{\phi} \phi-M \bar{\phi} \phi) \\
& +\frac{\lambda_{0}}{\lambda} \sqrt{|g|}\left(\frac { 1 } { 4 } \left[\mathrm{d}\left(\alpha_{m}-g_{m}\right)+\mathrm{d} \alpha_{m}-g_{m}\right.\right. \\
& \left.\quad-2 g_{m} v(\mathrm{~d}+1)\right] i \bar{\phi} \gamma^{m} \phi \\
+ & \frac{1}{4}\left[\lambda _ { 0 } \left(\mathcal{D}_{r m}-\partial_{m} g_{r}+\Gamma_{r m}^{n} g_{n}+\alpha_{m} g_{r}\right.\right. \\
& \left.\quad-\Delta_{m} g_{r}\right) g^{m r}+\frac{1}{\lambda_{0}}(\mathrm{~d}+1) \\
& \left.\left.+2 \frac{v(\mathrm{~d}+1)}{\lambda_{0}}\right] \bar{\phi} \gamma^{5} \phi\right) .
\end{aligned}
$$


A special choice of the weight $v=-\frac{1}{2}$ eliminates the induced chiral mass term (in the absence of gauge fields) and also eliminates the metric density contribution in the coupling to $i \bar{\phi} \gamma^{m} \phi$.

The only $\lambda$ dependence is in the overall coefficient $\frac{\lambda_{0}}{\lambda} \sqrt{|g|}$. As we will discuss in Sec. VIII, we may write $\ell \equiv \lambda / \lambda_{0}$ to be a dimensionless scale. By writing $\sqrt{|G|}=\frac{1}{\ell} \sqrt{|g|}$, we have

$$
\int d \lambda \frac{\lambda_{0}}{\lambda}=\lambda_{0} \int d \ell \frac{1}{\ell}=\lambda_{0} \log \left(\ell_{f} / \ell_{i}\right),
$$

where $\ell_{i}$ and $\ell_{f}$ are original and final length scales. With this, we can make a field redefinition of the fermions $\phi$ and define $\psi=\phi \sqrt{\lambda_{0} \log \left(\ell_{f} / \ell_{i}\right)}$ so that the fermions $\psi$ have the dimensions of four-dimensional fermions. The fourdimensional TW Dirac action becomes

$$
\begin{aligned}
S_{\mathrm{TWD}}= & \int d^{\mathrm{d}} x \sqrt{|g|}(i \bar{\psi} \not \mathbf{\psi} \psi-M \bar{\psi} \psi) \\
+ & +\int d^{\mathrm{d}} x \sqrt{|g|}\left(\frac { 1 } { 4 } \left[\mathrm{d}\left(\alpha_{m}-g_{m}\right)+\mathrm{d} \alpha_{m}-g_{m}\right.\right. \\
& \left.-2 g_{m} v(\mathrm{~d}+1)\right] i \bar{\psi} \gamma^{m} \psi \\
+ & \frac{1}{4}\left[\lambda_{0}\left(\mathcal{D}_{r m}-\partial_{m} g_{r}+\Gamma^{n}{ }_{r m} g_{n}+\alpha_{m} g_{r}-\Delta_{m} g_{r}\right) g^{m r}\right. \\
& \left.\left.+\frac{1}{\lambda_{0}}(\mathrm{~d}+1)+2 \frac{v(\mathrm{~d}+1)}{\lambda_{0}}\right] \bar{\psi} \gamma^{5} \psi\right) .
\end{aligned}
$$

We see that $\lambda_{0}$ still sets the chiral scale due to its presence in the last two summands of the action.

In the discussion following Eq. (75), we noted that we should expect a dynamical theory of $\mathcal{D}_{m n}$ to be sensitive to chirality of fermions. This expectation is realized by the TW Dirac Lagrangian, Eq. (135), due to the presence of $\gamma^{5}$. The theory is therefore chiral in this sense. We remark that one can still eliminate $\mathrm{d}$ degrees of freedom by using a coordinate gauge choice. For example, we could set $g_{a}=0$ (constant volume gauge for the metric), $\alpha_{a}=0$ (constant volume for the connection) or even $g_{a}=\alpha_{a}$ (compatibility of condition) in Eq. (142). However, no gauge choice will eliminate the $\mathcal{D}_{a b}$ fermion interaction.

\section{GAUGE INVARIANT TW ACTION}

The TW action was introduced in [20] in order to give dynamics to the diffeomorphism field. There, the correspondence with the coadjoint orbits of the Virasoro algebra was determined in the background of the gauged fixed 2D metric of Polyakov [31] that had constant volume. Similarly in [29], the interest was to study the diffeomorphism field as a primeval source for dark energy in a Friedman-Lemaitre-Robertson-Walker background in constant volume coordinates. As we have just seen in the Dirac action, writing the TW action in a gauge invariant form reveals physically interesting structure. From [20] the TW dynamical action is

$$
S=S_{\mathrm{PEH}}+S_{\mathrm{PGB}}
$$

where the projective Einstein-Hilbert action is

$$
S_{\mathrm{PEH}}=-\frac{1}{2 \tilde{\kappa}_{0} \lambda_{0}} \int d \lambda d^{\mathrm{d}} x \sqrt{|G|} \mathcal{K}^{a}{ }_{b c d}\left(\delta^{c}{ }_{a} g^{b d}\right)
$$

and the projective Gauss-Bonnet action is

$$
S_{\mathrm{PGB}}=-\frac{\tilde{J}_{0} c}{\lambda_{0}} \int d \lambda d^{\mathrm{d}} x \sqrt{|G|}\left(\mathcal{K}_{\beta \gamma \rho}^{\alpha} \mathcal{K}_{\alpha}^{\beta \gamma \rho}-4 \mathcal{K}_{\alpha \beta} \mathcal{K}^{\alpha \beta}+\mathcal{K}^{2}\right) .
$$

We remark that both terms are generalized Gauss-Bonnet terms and one could presumably continue adding generalized Gauss-Bonnet terms for higher interaction without compromising causality in the metric field equations [33]. Recall that the components of the TW curvature tensor $\mathcal{K}_{\beta \gamma \rho}^{\alpha}$ are given by

$$
\begin{aligned}
\mathcal{K}^{a}{ }_{b c d}= & \mathcal{R}^{a}{ }_{b c d}+\delta^{a}{ }_{c} \mathcal{D}_{d b}-\delta^{a}{ }_{d} \mathcal{D}_{c b} \\
= & R^{a}{ }_{b c d}+\delta^{a}{ }_{c} \mathcal{P}_{d b}-\delta^{a}{ }_{d} \mathcal{P}_{c b}-\delta^{a}{ }_{b} \mathcal{P}_{[c d]}, \\
\mathcal{K}_{b c d}^{\lambda}= & \lambda\left(\partial_{[c} \mathcal{D}_{d] b}+\Pi^{a}{ }_{b[d} \mathcal{D}_{c] a}\right) \\
= & \lambda\left(\partial_{[c} \mathcal{P}_{d] b}+\Gamma_{b[d}^{a} \mathcal{P}_{c] a}+\alpha_{[d} \mathcal{P}_{c] b}\right. \\
& \left.+\alpha_{b} \mathcal{P}_{[c d]}-R^{a}{ }_{b c d} \alpha_{a}\right), \\
\breve{\mathcal{K}}^{\lambda}{ }_{c a b} \equiv & \frac{1}{\lambda} \mathcal{K}^{\lambda}{ }_{c a b},
\end{aligned}
$$

where again

$$
\begin{aligned}
\alpha_{a} & =-\frac{1}{\mathrm{~d}+1} \Gamma_{e a}^{e}, \\
\Pi^{a}{ }_{b c} & =\Gamma^{a}{ }_{b c}+\delta^{a}{ }_{b} \alpha_{c}+\delta^{a}{ }_{c} \alpha_{b}, \\
\mathcal{R}^{a}{ }_{b c d} & =\partial_{c} \Pi^{a}{ }_{d b}-\partial_{d} \Pi^{a}{ }_{c b}+\Pi^{a}{ }_{c e} \Pi^{e}{ }_{d b}-\Pi^{a}{ }_{d e} \Pi^{e}{ }_{c b}, \\
R^{a}{ }_{b c d} & =\partial_{c} \Gamma^{a}{ }_{d b}-\partial_{d} \Gamma^{a}{ }_{c b}+\Gamma^{a}{ }_{c e} \Gamma^{e}{ }_{d b}-\Gamma^{a}{ }_{d e} \Gamma^{e}{ }_{c b}, \\
\mathcal{P}_{b c} & =\mathcal{D}_{b c}-\partial_{b} \alpha_{c}+\Gamma^{e}{ }_{b c} \alpha_{e}+\alpha_{b} \alpha_{c}
\end{aligned}
$$

for any affine connection $\Gamma_{b c}^{a}$. The nonzero components of the TW Ricci tensor $\mathcal{K}_{\alpha \beta}$ are

$\mathcal{K}_{b d}=\mathcal{R}_{b d}+(\mathrm{d}-1) \mathcal{D}_{b d}=R_{b d}+\mathrm{d} \mathcal{P}_{d b}-\mathcal{P}_{b d}$.

Then the projective Gauss-Bonnet action $S_{\mathrm{PGB}}$ may be decomposed as

$$
S_{\mathrm{PGB}}=S_{\mathrm{PGB} 1}+S_{\mathrm{PGB} 2}+S_{\mathrm{PGB} 3},
$$


where

$$
\begin{aligned}
S_{\mathrm{PGB} 1}= & -\frac{\tilde{J}_{0} c}{\lambda_{0}} \int d^{\mathrm{d}} x d \lambda \sqrt{|G|} \mathcal{K}^{a}{ }_{b c d} \mathcal{K}^{e}{ }_{f g h} \\
& \times\left(\mathcal{B}_{a e}{ }^{b f c g d h}-\lambda_{0}{ }^{2} g_{a} g_{e} g^{b f} g^{c g} g^{d h}\right), \\
S_{\mathrm{PGB} 2}= & \tilde{J}_{0} c \lambda_{0} \int d^{\mathrm{d}} x d \lambda \sqrt{|G|} \breve{\mathcal{K}}_{b c d} \breve{\mathcal{K}}_{f g h}\left(g^{b f} g^{c g} g^{d h}\right), \\
S_{\mathrm{PGB} 3}= & 2 \tilde{J}_{0} c \lambda_{0} \int d^{\mathrm{d}} x d \lambda \sqrt{|G|} \mathcal{K}^{a}{ }_{b c d} \breve{\mathcal{K}}_{f g h}\left(g_{a} g^{b f} g^{c g} g^{d h}\right),
\end{aligned}
$$

and we have defined the Gauss-Bonnet operator as

$$
\begin{aligned}
\mathcal{G}_{\alpha \bar{\alpha}}{ }^{\beta \bar{\beta} \gamma \bar{\gamma} \rho \bar{\rho}}= & G_{\alpha \bar{\alpha}} G^{\beta \bar{\beta}} G^{\gamma \bar{\gamma}} G^{\rho \bar{\rho}}-4 \delta^{\gamma}{ }_{\alpha} \delta^{\bar{\gamma}} G_{\bar{\alpha}}^{\beta \bar{\beta}} G^{\rho \bar{\rho}} \\
& +\delta^{\gamma}{ }_{\alpha} \delta^{\bar{\gamma}} G^{\beta \rho} G^{\bar{\beta} \bar{\rho}}
\end{aligned}
$$

and for convenience, in terms of the metric on $\mathcal{M}$,

$$
\begin{aligned}
\mathcal{B}_{a \bar{a}} b \bar{b} g \bar{g} r \bar{r} & =g_{a \bar{a}} g^{b \bar{b}} g^{g \bar{g}} g^{r \bar{r}}-4 \delta^{g}{ }_{a} \delta^{\bar{g}} \bar{a} g^{b \bar{b}} g^{r \bar{r}} \\
& +\delta^{g}{ }_{a} \delta^{\bar{g}}{ }^{\bar{a}} g^{b r} g^{\bar{b} \bar{r}} .
\end{aligned}
$$

Finally, we can write the full dynamical action as

$$
S=S_{\mathrm{PEH}}+S_{\mathrm{PGB} 1}+S_{\mathrm{PGB} 2}+S_{\mathrm{PGB} 3} .
$$

This form of the action is convenient for computing field equations. The curvature components $\mathcal{K}^{a}{ }_{b c d}$ and $\breve{\mathcal{K}}_{b c d}$ carry all of the $\Pi_{b c}^{a}$ and $\mathcal{D}_{b c}$ (equivalently $\Gamma_{b c}^{a}$ and $\mathcal{P}_{b c}$ ) dependence, while the metric tensor $g_{a b}$ appears elsewhere in each part of the action, including in the Gauss-Bonnet operator $\mathcal{B}$.

To illustrate explicit general coordinate invariance, it is also possible to decompose the action as

$$
\begin{aligned}
S= & \left(\int \frac{1}{\lambda} d \lambda\right)\left[-\frac{1}{2 \tilde{\mathcal{K}}_{0}} \int d^{\mathrm{d}} x \sqrt{|g|} \mathcal{K}-\tilde{J}_{0} c \int d^{\mathrm{d}} x \sqrt{|g|}\left(\mathcal{K}^{a}{ }_{b c d} \mathcal{K}_{a}{ }^{b c d}-4 \mathcal{K}_{a b} \mathcal{K}^{a b}+\mathcal{K}^{2}\right)\right. \\
& +\tilde{J}_{0} c \lambda_{0}^{2} \int d^{\mathrm{d}} x \sqrt{|g|} \underbrace{\left(g_{a} \mathcal{K}^{a}{ }_{b c d}+\breve{\mathcal{K}}_{b c d}\right)}_{\text {tensor }} \underbrace{\left(g_{e} \mathcal{K}^{e}{ }_{f g h}+\breve{\mathcal{K}}_{f g h}\right)}_{\text {tensor }} g^{b f} g^{c g} g^{d h}] .
\end{aligned}
$$

Equations (146) and (148) demonstrate that $\mathcal{K}^{a}{ }_{b c d}, \mathcal{K}_{a b}$, and $\mathcal{K}$ are tensors on the spacetime manifold $\mathcal{M}$. Furthermore, we introduce $K_{b c d} \equiv \mathcal{K}_{b c d}(g)$ as the following rank-three tensor on $\mathcal{M}$

$$
\begin{aligned}
K_{b c d} \equiv & g_{a} \mathcal{K}^{a}{ }_{b c d}+\breve{\mathcal{K}}_{b c d} \\
= & \left(g_{a}-\alpha_{a}\right) R^{a}{ }_{b c d}+\left(g_{c}-\alpha_{c}\right) \mathcal{P}_{d b}-\left(g_{d}-\alpha_{d}\right) \mathcal{P}_{c b} \\
& -\left(g_{b}-\alpha_{b}\right) \mathcal{P}_{[c d]}+\nabla_{c} \mathcal{P}_{d b}-\nabla_{d} \mathcal{P}_{c b},
\end{aligned}
$$

where $\nabla_{a}$ is the covariant derivative operator associated with the spacetime connection $\Gamma_{b c}^{a}$. Since $g_{a}$ and $\alpha_{a}$ have the same coordinate transformation law, we see that $K_{b c d}$ is indeed a tensor on $\mathcal{M}$. This demonstrates that the action is a scalar as well as projectively invariant.

Owing to Eq. (154), all the $\lambda$ dependence appears as overall coefficients. We will use the interpretation of the coupling constants as in [29] to write them in terms of scale dependent quantities. Let $\ell \equiv \lambda / \lambda_{0}$ be a dimensionless scale. Since only $\int d \lambda \frac{1}{\lambda}$ appears in the overall coupling, we again write $\sqrt{|G|}=\frac{1}{\ell} \sqrt{|g|}$. Then by integrating over $\ell$, we can rewrite the action in terms of coupling constants that have familiar interpretations

$\frac{1}{\tilde{\kappa}_{0}} \int_{\ell_{i}}^{\ell_{f}} d \ell \frac{1}{\ell}=\frac{\log \left(\ell_{f} / \ell_{i}\right)}{\tilde{\kappa}_{0}} \Rightarrow \kappa_{0} \equiv \frac{\tilde{\kappa_{0}}}{\log \left(\ell_{f} / \ell_{i}\right)}$,
$\tilde{J}_{0} \int_{\ell_{i}}^{\ell_{f}} d \ell \frac{1}{\ell}=\tilde{J}_{0} \log \left(\ell_{f} / \ell_{i}\right) \Rightarrow J_{0} \equiv \tilde{J}_{0} \log \left(\ell_{f} / \ell_{i}\right)$.

Thus a natural scaling of the gravitational coupling constant $\kappa_{0}$ and angular momentum parameter $J_{0}$ occurs as we move from one length scale to another. In this way, projective geometry has a potential renormalization group interpretation. This link is under further investigation. The characteristic projective length scale (inverse mass scale) is set by $\lambda_{0}$. With this, we can rewrite the TW action as

$$
\begin{aligned}
S= & -\frac{1}{2 \kappa_{0}} \int d^{\mathrm{d}} x \sqrt{|g|} \mathcal{K}+c J_{0} \lambda_{0}{ }^{2} \int d^{\mathrm{d}} x \sqrt{|g|} K_{b c d} K^{b c d} \\
& -c J_{0} \int d^{\mathrm{d}} x \sqrt{|g|}\left(\mathcal{K}^{a}{ }_{b c d} \mathcal{K}_{a}{ }^{b c d}-4 \mathcal{K}_{a b} \mathcal{K}^{a b}+\mathcal{K}^{2}\right)
\end{aligned}
$$

where $K^{b c d}$ and $\mathcal{K}_{a}{ }^{b c d}$ have had the altitudes of their indices flipped via the metric and inverse metric on $\mathcal{M}$

$$
\begin{aligned}
K^{b c d} & =g^{b f} g^{c g} g^{d h} K_{f g h}, \\
\mathcal{K}_{a}{ }^{b c d} & =g_{a m} g^{b f} g^{c g} g^{d h} \mathcal{K}^{m}{ }_{f g h} .
\end{aligned}
$$




\section{THE COVARIANT FIELD EQUATIONS}

In the spirit of Palatini [32], we will treat the metric tensor $g_{a b}$ and $\tilde{\Gamma}_{\beta \gamma}^{\alpha}$ as independent degrees of freedom. This fits the framework of TW gravity, since the TW connection is to be thought of as a connection over the space of equivalence classes of connections and is not naturally tied to a particular metric. The metric $G_{\mu \nu}$ serves only to maintain general coordinate invariance on $\mathcal{N}$, just as $\mathcal{D}_{a b}$ exists in order to make the connection $\tilde{\nabla}_{\mu}$ covariant. The covariant derivative is a projective invariant that is constructed only from projectively invariant quantities such as $\Pi_{b c}^{a}$ and $\lambda$. However, as one sees in Eq. (22), the only degrees of freedom that are allowed to fluctuate are $\mathcal{D}_{a b}$ and $\Pi^{a}{ }_{b c}$. Therefore we will only need the field equations for $\Pi_{b c}^{a}, \mathcal{D}_{a b}$, and $g_{a b}$. We note that $\lambda$ does not fluctuate and only sets the volume scale.

We consider a total action of the form

$$
S_{\text {total }}=S+S_{\text {matter }},
$$

where here $S$ is the TW action from Eq. (143) and $S_{\text {matter }}$ are contributions from other sources. For example the Dirac action for each species of fermions will be in the form of
Eq. (142) and could be accompanied by an appropriate gauge field action for Yang-Mills fields. Other matter contributions may also be considered. In what follows, however, we will derive the field equations from $S$ only with the understanding that the matter actions will also contribute nontrivially to these equations. For the field equations of the metric, Sec. IX C, we will reinstate the matter contribution through the energy-momentum tensor, $\Theta_{p q}^{\text {matter }}$. In Sec. IX D we will demonstrate how one may use a Palatini field, $C^{a}{ }_{b c}$, to utilize a metric compatible connection and recover the usual Einstein field equations with a divergence free energy-momentum tensor. All the field equations in this section will be summarized in the Appendix B.

\section{A. Equations of motion for $\Pi_{b c}^{a}$}

In order to simplify the computation of the field equations, we will use $\mathcal{F}$ to denote an object with the correct valence to form a scalar with another given object. For example, we might write an expression such as $\mathcal{K}^{a}{ }_{b c d} \mathcal{F}$, where we would understand that $\mathcal{F}$ is an object with components $\mathcal{F}_{a}{ }^{b c d}$ such that $\mathcal{F}$ forms a scalar upon tensor multiplication with $\mathcal{K}^{a}{ }_{b c d}$.

With this, we compute the field equations for $\Pi_{b c}^{a}$ as

$$
\begin{aligned}
S= & \int \mathcal{K}^{a}{ }_{b c d} \mathcal{F} \\
\Rightarrow \delta S= & \int\left(\delta \mathcal{K}^{a}{ }_{b c d}\right) \mathcal{F} \\
= & \int \delta\left(\mathcal{R}^{a}{ }_{b c d}+\delta^{a}{ }_{c} \mathcal{D}_{d b}-\delta^{a}{ }_{d} \mathcal{D}_{c b}\right) \mathcal{F} \\
= & \int \delta\left(\partial_{c} \Pi^{a}{ }_{d b}-\partial_{d} \Pi^{a}{ }_{c b}+\Pi^{a}{ }_{c e} \Pi^{e}{ }_{d b}-\Pi^{a}{ }_{d e} \Pi^{e}{ }_{c b}\right) \mathcal{F} \\
= & \int\left(-\delta^{a}{ }_{l} \delta^{m}{ }_{d} \delta^{n}{ }_{b} \partial_{c} \mathcal{F}+\delta^{a}{ }_{l} \delta^{m}{ }_{c} \delta^{n}{ }_{b} \partial_{d} \mathcal{F}+\left(\delta^{a}{ }_{l} \delta^{m}{ }_{c} \delta^{n}{ }_{e} \Pi^{e}{ }_{d b}+\delta^{e}{ }_{l} \delta^{m}{ }_{d} \delta^{n}{ }_{b} \Pi^{a}{ }_{c e}-\delta^{a}{ }_{l} \delta^{m}{ }_{d} \delta^{n}{ }_{e} \Pi^{e}{ }_{c b}\right.\right. \\
& \left.\left.-\delta^{e}{ }_{l} \delta^{m}{ }_{c} \delta^{n}{ }_{b} \Pi^{a}{ }_{d e}\right) \mathcal{F}\right) \delta \Pi^{l}{ }_{m n}
\end{aligned}
$$

and

$$
\begin{aligned}
S & =\int \breve{\mathcal{K}}_{c a b} \mathcal{F} \\
\Rightarrow \delta S & =\int\left(\delta \breve{\mathcal{K}}_{c a b}\right) \mathcal{F} \\
& =\int \delta\left(\partial_{[a} \mathcal{D}_{b] c}+\Pi^{d}{ }_{c[b} \mathcal{D}_{a] d}\right) \mathcal{F} \\
& =\int\left(\left(\delta^{d}{ }_{l} \delta^{m}{ }_{c} \delta^{n}{ }_{b} \mathcal{D}_{a d}-\delta^{d}{ }_{l} \delta^{m}{ }_{c} \delta^{n}{ }_{a} \mathcal{D}_{b d}\right) \mathcal{F}\right) \delta \Pi^{l}{ }_{m n} .
\end{aligned}
$$

These two variations lead to the full equations of motion for $\Pi_{b c}^{a}$ that are associated with the appropriate object $\mathcal{F}$. We have 


$$
\begin{aligned}
\delta S_{\mathrm{PEH}}= & -\frac{1}{2 \kappa_{0}} \int d^{\mathrm{d}} x\left[-\partial_{l}\left(\sqrt{|g|} g^{n m}\right)+\delta^{m}{ } \partial_{e}\left(\sqrt{|g| g^{n e}}\right)\right. \\
& +\sqrt{|g|}\left(\delta^{m} \mathrm{H}_{d b}^{n g^{b d}}+\Pi^{a}{ }_{a l} g^{n m}\right. \\
& \left.\left.-\Pi^{n}{ }_{l b} g^{b m}-\Pi^{m}{ }_{d l} g^{n d}\right)\right] \delta \Pi^{l}{ }_{m n},
\end{aligned}
$$

where the striked-out terms vanish because $\Pi_{b c}^{a}$ as well as its variation $\delta \Pi^{a}{ }_{b c}$ are traceless. The remaining contribution to the field equations would vanish if $\Pi_{b c}^{a}$ were the traceless Levi-Civita connection of the metric $g_{a b}$, consistent with the original Palatini equations [32]. The next contributions are

$$
\begin{aligned}
& \delta S_{\mathrm{PGB} 1}=-2 J_{0} c \int d^{\mathrm{d}} x\left[\partial_{e}\left(\sqrt{|g|} \mathcal{K}^{a}{ }_{b c d}\left(\mathcal{B}_{l a}{ }^{n b m c e d}-\mathcal{B}_{l a}{ }^{n b e c m d}+\lambda_{0}{ }^{2} g_{l} g_{a} g^{n b} g^{e c} g^{m d}-\lambda_{0}^{2} g_{l} g_{a} g^{n b} g^{m c} g^{e d}\right)\right)\right. \\
&++\sqrt{|g|} \mathcal{K}^{a}{ }_{b c d}\left(\Pi^{n}{ }_{e f}\left(\mathcal{B}_{l a}{ }^{f b m c e d}-\mathcal{B}_{l a}{ }^{f b e c m d}+\lambda_{0}{ }^{2} g_{l} g_{a} g^{f b} g^{e c} g^{m d}-\lambda_{0}{ }^{2} g_{l} g_{a} g^{f b} g^{m c} g^{e d}\right)\right. \\
&+\left.\left.\Pi^{e}{ }_{f l}\left(\mathcal{B}_{e a}{ }^{n b f c m d}-\mathcal{B}_{e a}{ }^{n b m c f d}+\lambda_{0}{ }^{2} g_{e} g_{a} g^{n b} g^{m c} g^{f d}-\lambda_{0}{ }^{2} g_{e} g_{a} g^{n b} g^{f c} g^{m d}\right)\right)\right] \delta \Pi^{l}{ }_{m n}, \\
& \delta S_{\mathrm{PGB} 2}=4 J_{0} c \lambda_{0}{ }^{2} \int d^{\mathrm{d}} x\left[\sqrt{|g|} \breve{\mathcal{K}}_{f g h} \mathcal{D}_{c l} g^{m f} g^{c g} g^{n h}\right] \delta \Pi^{l}{ }_{m n}, \\
& \delta S_{\mathrm{PGB} 3}= 4 J_{0} c \lambda_{0}{ }^{2} \int d^{\mathrm{d}} x\left[\partial_{d}\left(\sqrt{|g|} \breve{\mathcal{K}}_{f g h} g_{l} g^{n f} g^{m g} g^{d h}\right)+\sqrt{|g|} \breve{\mathcal{K}}_{f g h}\left(g_{l} g^{b f} g^{m g} g^{d h} \Pi^{n}{ }_{d b}+g_{a} g^{n f} g^{c g} g^{m h} \Pi^{a}{ }_{c l}\right)\right. \\
&\left.+\sqrt{|g|} \mathcal{K}^{a}{ }_{b c d} g_{a} g^{b m} g^{c g} g^{d n} \mathcal{D}_{g l}\right] \delta \Pi^{l}{ }_{m n} .
\end{aligned}
$$

By defining

$$
\hat{\mathcal{K}}_{a}{ }^{b g r}=\mathcal{K}_{\bar{b}}^{\bar{b} \bar{g} \bar{r}} \mathcal{G}_{a \bar{a}}{ }^{b \bar{b}}[g|\bar{g}| r] \bar{r} \quad \text { and } \quad g_{\beta}=\left(g_{b}, \frac{1}{\lambda}\right),
$$

where the sums are restricted to $\mathcal{M}$ coordinates, the variation can be written succinctly as

$$
\begin{aligned}
\delta S=0 & =\int d^{\mathrm{d}} x \sqrt{|g|}\left[E_{a}{ }^{m n}-\frac{1}{\mathrm{~d}+1} \delta_{a}{ }^{(m} E_{b}{ }^{n) b}\right] \delta \Pi^{a}{ }_{m n}, \\
E_{a}{ }^{m n}= & E_{a}{ }^{n m}=\frac{1}{2 \kappa_{0} J_{0} c} \breve{\nabla}_{a}\left(\sqrt{|g|} g^{m n}\right)-\breve{\nabla}_{c}\left(\sqrt{|g|} \hat{\mathcal{K}}_{a}{ }^{(m n) c}\right) \\
& +2 \lambda_{0}^{2} \breve{\nabla}_{c}\left(\sqrt{|g|} g_{a} \breve{\mathcal{K}}^{(m n) c}\right) \\
& -2 \lambda_{0}^{2} \sqrt{|g|} K^{(m n) c} \mathcal{D}_{c a} .
\end{aligned}
$$

Here, $\breve{\nabla}_{a}$ is the derivative operator with respect to the fundamental projective invariant $\Pi_{m n}^{l}$ with action as follows:

$$
\breve{\nabla}_{d} \sqrt{|g|}=\partial_{d} \sqrt{|g|}-\Pi_{a d}^{a} \sqrt{|g|}=\partial_{d} \sqrt{|g|}
$$

$$
\begin{aligned}
\breve{\nabla}_{d} & \left.\sqrt{|g|} \hat{\mathcal{K}}_{a}{ }^{m n c}\right) \\
= & \left(\partial_{d} \sqrt{|g|}\right) \hat{\mathcal{K}}_{a}{ }^{m n c}+\sqrt{|g|} \breve{\nabla}_{d} \hat{\mathcal{K}}_{a}{ }^{m n c} \\
= & \left(\partial_{d} \sqrt{|g|}\right) \hat{\mathcal{K}}_{a}{ }^{m n c}+\sqrt{|g|}\left(\partial_{d} \hat{\mathcal{K}}_{a}{ }^{m n c}-\Pi^{f}{ }_{a d} \hat{\mathcal{K}}_{f}^{m n c}\right) \\
& +\sqrt{|g|}\left(\Pi^{m}{ }_{f d} \hat{\mathcal{K}}_{a}{ }^{f n c}+\Pi^{n}{ }_{f d} \hat{\mathcal{K}}_{a}^{m f c}+\Pi^{c}{ }_{f d} \hat{\mathcal{K}}_{a}{ }^{m n f}\right) .
\end{aligned}
$$

Thus, the field equations for $\Pi^{a}{ }_{m n}$ are

$$
E_{a}{ }^{m n}-\frac{1}{\mathrm{~d}+1} \delta_{a}^{(m} E_{b}{ }^{n) b}=0 .
$$

We note that if the connection were chosen to be compatible with the metric $g_{a b}$, then in the language of tractor calculus [28], Eq. (171) would imply that the projective curvature is Yang-Mills [26].

\section{B. Equations of motion for $\mathcal{D}_{b c}$}

To find the field equations for $\mathcal{D}_{b c}$, we proceed in the same manner as we did for $\Pi_{b c}^{a}$. The contributions are of the form 


$$
\begin{aligned}
S & =\int \mathcal{K}^{a}{ }_{b c d} \mathcal{F} \\
\Rightarrow \delta S & =\int\left(\delta \mathcal{K}^{a}{ }_{b c d}\right) \mathcal{F} \\
& =\int \delta\left(\mathcal{R}^{a}{ }_{b c d}+\delta^{a}{ }_{c} \mathcal{D}_{d b}-\delta^{a}{ }_{d} \mathcal{D}_{c b}\right) \mathcal{F} \\
& =\int\left[\left(\delta^{a}{ }_{c} \delta^{p}{ }_{d} \delta^{q}{ }_{b}-\delta^{a}{ }_{d} \delta^{p}{ }_{c} \delta^{q}{ }_{b}\right) \mathcal{F}\right] \delta \mathcal{D}_{p q}
\end{aligned}
$$

and

$$
\begin{aligned}
S= & \int \breve{\mathcal{K}}_{b c d} \mathcal{F} \\
\Rightarrow \delta S= & \int\left(\delta \breve{\mathcal{K}}_{b c d}\right) \mathcal{F} \\
= & \int \delta\left(\partial_{[c} \mathcal{D}_{d] b}+\Pi^{e}{ }_{b[d} \mathcal{D}_{c] e}\right) \mathcal{F} \\
= & \int\left[-\delta^{p}{ }_{d} \delta^{q}{ }_{b} \partial_{c} \mathcal{F}+\delta^{p}{ }_{c} \delta^{q}{ }_{b} \partial_{d} \mathcal{F}\right. \\
& \left.+\left(\Pi^{q}{ }_{b d} \delta^{p}{ }_{c}-\Pi^{q}{ }_{b c} \delta^{p}{ }_{d}\right) \mathcal{F}\right] \delta \mathcal{D}_{p q} .
\end{aligned}
$$

Again, by assigning the appropriate object $\mathcal{F}$ to each term we have

$$
\begin{aligned}
& \delta S_{\mathrm{PEH}}=-\frac{1}{2 \kappa_{0}} \int d^{\mathrm{d}} x \sqrt{|g|}\left[(\mathrm{d}-1) g^{q p}\right] \delta \mathcal{D}_{p q}, \\
& \delta S_{\mathrm{PGB} 1}=-2 J_{0} c \int d^{\mathrm{d}} x \sqrt{|g|} \mathcal{K}^{e}{ }_{f g h}\left[\mathcal{B}_{c e^{q f c g p h}}-\mathcal{B}_{c e^{q f p g c h}}\right. \\
& \left.+2 \lambda_{0}^{2} g_{c} g_{e} g^{q f} g^{p g} g^{c h}\right] \delta \mathcal{D}_{p q}, \\
& \delta S_{\mathrm{PGB} 2}=4 J_{0} c \lambda_{0}^{2} \int d^{d} x\left[\partial_{c}\left(\sqrt{|g|} \breve{\mathcal{K}}_{f g h} g^{q f} g^{p g} g^{c h}\right)\right. \\
& \left.+\sqrt{|g|} \breve{\mathcal{K}}_{f g h} \Pi_{b c}^{q} g^{b f} g^{p g} g^{c h}\right] \delta \mathcal{D}_{p q}, \\
& \delta S_{\mathrm{PGB} 3}=4 J_{0} \lambda_{0}^{2} \int d^{\mathrm{d}} x\left[\sqrt{|g|} \breve{\mathcal{K}}_{f g h} g_{a} g^{q f} g^{a g} g^{p h}\right. \\
& +\partial_{g}\left(\sqrt{|g|} \mathcal{K}^{a}{ }_{b c d} g_{a} g^{b q} g^{c p} g^{d g}\right) \\
& \left.+\sqrt{|g|} \mathcal{K}_{b c d}^{a} g_{a} g^{b f} g^{c p} g^{d g} \Pi_{f g}^{q}\right] \delta \mathcal{D}_{p q} .
\end{aligned}
$$

Then the variation with respect to $\delta \mathcal{D}_{p q}$ yields

$$
\begin{aligned}
- & \frac{1}{2 \kappa_{0} J_{0} c} \sqrt{|g|}(\mathrm{d}-1) g^{p q}+\sqrt{|g|} \hat{\mathcal{K}}_{c}{ }^{(p q) c} \\
& +2 \lambda_{0}^{2} \breve{\nabla}_{g}\left(\sqrt{|g|} K^{(p q) g}\right)-2 \lambda_{0}^{2} \sqrt{|g|} g_{c} \breve{\mathcal{K}}^{(p q) c}=0 .
\end{aligned}
$$

Note the derivative of $K^{(p q) g}$, which makes the field equations second-order differential equations in $\mathcal{D}_{b c}$.

\section{Equations of motion for $g_{b c}$}

Finally, we will find the field equations for the spacetime metric tensor $g_{b c}$. For the sake of familiarity, we will write the TW action $S$, Eq. (157), as

$$
S=-\frac{1}{2 \kappa_{0}} \int d^{\mathrm{d}} x \sqrt{|g|} g^{a b} R(\Gamma)_{a b}+\int d^{\mathrm{d}} x \sqrt{|g|} \mathcal{L}_{\mathrm{S}}
$$

so as to separate the Einstein-Hilbert-Palatini action from the rest of the action. The $\mathcal{L}_{\mathrm{S}}$ is the remaining part of the Lagrangian density on $\mathcal{M}$, viz.,

$$
\begin{aligned}
\mathcal{L}_{\mathrm{S}}= & -\frac{1}{2 \kappa_{0}}(\mathrm{~d}-1) \mathcal{P}+c J_{0} \lambda_{0}{ }^{2} K_{b c d} K^{b c d} \\
& -c J_{0}\left(\mathcal{K}^{a}{ }_{b c d} \mathcal{K}_{a}{ }^{b c d}-4 \mathcal{K}_{a b} \mathcal{K}^{a b}+\mathcal{K}^{2}\right) .
\end{aligned}
$$

We have explicitly written the Ricci tensor as $R(\Gamma)_{a b}$ to emphasize the independence of the connection from the metric. In what follows we write $R \equiv R(\Gamma)_{a b} g^{a b}$ and $R_{a b} \equiv R(\Gamma)_{a b}$. The total action that contains the TW action and any matter fields is written as

$$
\begin{aligned}
S_{\text {total }}= & -\frac{1}{2 \kappa_{0}} \int d^{\mathrm{d}} x \sqrt{|g|} g^{a b} R_{a b}+\int d^{\mathrm{d}} x \sqrt{|g|} \mathcal{L}_{\mathrm{S}} \\
& +\int d^{\mathrm{d}} x \sqrt{|g|} \mathcal{L}_{\text {matter }} .
\end{aligned}
$$

We can define energy-momentum tensors $\Theta_{p q}^{\mathrm{S}}$ and $\Theta_{p q}^{\text {matter }}$ from the variation of the action with respect to the inverse metric $g^{p q}$, via

$$
\begin{aligned}
\delta S_{\text {total }} & =\int \sqrt{|g|} d^{\mathrm{d}} x\left(\frac{1}{\sqrt{|g|}} \frac{\delta\left(\sqrt{|g|} \mathcal{L}_{\mathrm{S}}\right)}{\delta g^{p q}}+\frac{1}{\sqrt{|g|}} \frac{\delta\left(\sqrt{|g|} \mathcal{L}_{\text {matter }}\right)}{\delta g^{p q}}\right. \\
& \left.-\frac{1}{2 \kappa_{0}}\left(\frac{\delta R}{\delta g^{p q}}+\frac{R}{\sqrt{|g|}} \frac{\delta \sqrt{|g|}}{\delta g^{p q}}\right)\right) \delta g^{p q}=0 .
\end{aligned}
$$

Then from the Einstein-Palatini equations,

$$
\frac{1}{2} R_{(p q)}-\frac{1}{2} R g_{p q}=\kappa_{0}\left(\Theta_{p q}^{\mathrm{S}}+\Theta_{p q}^{\mathrm{matter}}\right),
$$

gives the energy-momentum tensors defined as

$$
\begin{aligned}
\Theta_{p q}^{\mathrm{S}} & =\frac{2}{\sqrt{|g|}} \frac{\delta\left(\sqrt{|g|} \mathcal{L}_{\mathrm{S}}\right)}{\delta g^{p q}} \text { and } \\
\Theta_{p q}^{\text {matter }} & =\frac{2}{\sqrt{|g|}} \frac{\delta\left(\sqrt{|g|} \mathcal{L}_{\text {matter }}\right)}{\delta g^{p q}},
\end{aligned}
$$

with $\kappa_{0}=\frac{8 \pi G}{c^{4}}$. Because the connection is not compatible with the metric, the left- and right-hand sides of Eq. (183) are not separately divergence free. We will address this in the next subsection. 
More precisely, the energy-momentum tensor, $\Theta_{p q}^{\mathrm{S}}$, arises from $S$, by first exposing the Einstein-Palatini tensor from the action which resides in the $S_{\mathrm{PEH}}$ summand of $S$. It has the variation

$$
\begin{aligned}
\delta S_{\mathrm{PEH}} & =-\frac{1}{2 \kappa_{0}} \int d^{\mathrm{d}} x \mathcal{K}^{a}{ }_{b c d} \delta^{c}{ }_{a} \delta\left(\sqrt{|g|} g^{b d}\right) \\
& =-\frac{1}{2 \kappa_{0}} \int d^{\mathrm{d}} x \sqrt{|g|} \mathcal{K}^{a}{ }_{b c d} \delta^{c}{ }_{a}\left(\delta^{b}{ }_{i} \delta^{d}{ }_{j}-\frac{1}{2} g_{i j} g^{b d}\right)\left(\delta g^{i j}\right) \\
& =-\frac{1}{2 \kappa_{0}} \int d^{\mathrm{d}} x \sqrt{|g|}\left(\mathcal{K}_{i j}-\frac{1}{2} g_{i j} \mathcal{K}\right)\left(\delta g^{i j}\right) \\
& =-\frac{1}{2 \kappa_{0}} \int d^{\mathrm{d}} x \sqrt{|g|}\left(\left(R_{i j}-\frac{1}{2} g_{i j} R\right)\right. \\
& \left.-(\mathrm{d}-1)\left(\mathcal{P}_{i j}-\frac{1}{2} \mathcal{P} g_{i j}\right)\right)\left(\delta g^{i j}\right)
\end{aligned}
$$

where the Einstein-Palatini tensor is easily recognized. Continuing to the $S_{\mathrm{PGB} 1}$ term, we first find

$$
\begin{aligned}
\delta( & \left.\sqrt{|g|} \mathcal{B}_{a e}{ }^{b f c g d h}\right) \mathcal{F}=\int \sqrt{|g|}\left[-\frac{1}{2} g_{i j} \mathcal{B}_{a e}{ }^{b f c g d h}\right. \\
& +\delta^{c}{ }_{a} \delta^{g}{ }_{e} \delta^{b}{ }_{i} \delta^{d}{ }_{j} g^{f h}+\delta^{c}{ }_{a} \delta^{g}{ }_{e} \delta^{f} \delta^{d}{ }_{j} g^{b d} \\
& -4 \delta^{c}{ }_{a} \delta^{g}{ }_{e} \delta^{b}{ }_{i} \delta^{f}{ }_{j} g^{d h}-4 \delta^{c}{ }_{a} \delta^{g}{ }_{e} \delta^{d}{ }_{i} \delta^{h}{ }_{j} g^{b f} \\
& -g_{a i} g_{e j} g^{b f} g^{c g} g^{d h}+g_{a e} \delta^{b}{ }_{i} \delta^{f}{ }_{j} g^{c g} g^{d h} \\
& \left.+g_{a e} \delta^{c}{ }_{i} \delta^{g}{ }_{j} g^{b f} g^{d h}+g_{a e} \delta^{d}{ }_{i} \delta^{h}{ }_{j} g^{b f} g^{c g}\right] \mathcal{F}\left(\delta g^{i j}\right) .
\end{aligned}
$$

We can get part of the variation of $S_{\mathrm{PGB} 1}$ by putting a constant in front of Eq. (186) and plugging in the appropriate $\mathcal{F}=\mathcal{K}^{a}{ }_{b c d} \mathcal{K}^{e}{ }_{f g h}$. The other part of the variation of $S_{\mathrm{PGB} 1}$ can be found separately. Altogether, we have

$$
\begin{aligned}
\delta S_{\mathrm{PGB} 1}= & -J_{0} c \int d^{\mathrm{d}} x \sqrt{|g|}\left[-\frac{1}{2} g_{i j} \mathcal{B}_{a e}{ }^{b f c g d h} \mathcal{K}^{a}{ }_{b c d} \mathcal{K}^{e}{ }_{f g h}\right. \\
& +2 \mathcal{K}_{i j} \mathcal{K}-8 \mathcal{K}_{i b} \mathcal{K}_{j d} g^{b d}-\mathcal{K}_{i a b d} \mathcal{K}_{j}{ }^{a d b}+\mathcal{K}^{c}{ }_{i d b} \mathcal{K}_{c j}{ }^{d b}+\mathcal{K}^{c a}{ }_{i b} \mathcal{K}_{c a j}{ }^{b}+\mathcal{K}_{i}^{c a d} \mathcal{K}_{c a d j} \\
& +\lambda_{0}{ }^{2}\left\{\frac{1}{\mathrm{~d}+1} g_{i j} \partial_{a}\left(g_{e} g^{b f} g^{c g} g^{d h} \mathcal{K}^{a}{ }_{b c d} \mathcal{K}^{e}{ }_{f g h}\right)\right. \\
& \left.\left.-g_{a} g_{e} g^{c g} g^{d h}\left(\frac{1}{2} g^{b f} g_{i j} \mathcal{K}^{a}{ }_{b c d} \mathcal{K}^{e}{ }_{f g h}+\mathcal{K}^{a}{ }_{i c d} \mathcal{K}^{e}{ }_{j g h}+\mathcal{K}^{a}{ }_{c d i} \mathcal{K}^{e}{ }_{g h j}+\mathcal{K}^{a}{ }_{d i c} \mathcal{K}^{e}{ }_{h j g}\right)\right\}\right]\left(\delta g^{i j}\right) .
\end{aligned}
$$

Similarly, variation of $S_{\mathrm{PGB} 2}$ is given by

$$
\begin{aligned}
\delta S_{\mathrm{PGB} 2} & =J_{0} \lambda_{0}{ }^{2} \int d^{\mathrm{d}} x \delta\left(\sqrt{|g|} g^{b f} g^{c g} g^{d h}\right) \breve{\mathcal{K}}_{b c d} \breve{\mathcal{K}}_{f g h} \\
& =J_{0} \lambda_{0}{ }^{2} \int d^{\mathrm{d}} x \sqrt{|g|}\left[\frac{1}{2} g_{i j} g^{b f} g^{c g} g^{d h}+\delta^{c}{ }_{i} \delta^{g}{ }_{j} g^{d h} g^{b f}+\delta^{d}{ }_{i} \delta^{h}{ }_{j} g^{c g} g^{b f}+\delta^{b}{ }_{i} \delta^{f}{ }_{j} g^{c g} g^{d h}\right] \breve{\mathcal{K}}_{b c d} \breve{\mathcal{K}}_{f g h}\left(\delta g^{i j}\right) \\
& =J_{0} \lambda_{0}{ }^{2} \int d^{\mathrm{d}} x \sqrt{|g|} g^{c g} g^{d h}\left(\frac{1}{2} g^{b f} g_{i j} \breve{\mathcal{K}}_{b c d} \breve{\mathcal{K}}_{f g h}+\breve{\mathcal{K}}_{c d i} \breve{\mathcal{K}}_{g h j}+\breve{\mathcal{K}}_{d i c} \breve{\mathcal{K}}_{h j g}+\breve{\mathcal{K}}_{i c d} \breve{\mathcal{K}}_{j g h}\right)\left(\delta g^{i j}\right) .
\end{aligned}
$$

Finally, the variation of $S_{\mathrm{PGB} 3}$ is given by

$$
\begin{aligned}
\delta S_{\mathrm{PGB} 3} & =2 J_{0} \lambda_{0}{ }^{2} \int d^{\mathrm{d}} x \delta\left(\sqrt{|g|} g_{a} g^{b f} g^{c g} g^{d h}\right) \mathcal{K}^{a}{ }_{b c d} \breve{\mathcal{K}}_{f g h} \\
& =2 J_{0} \lambda_{0}{ }^{2} \int d^{\mathrm{d}} x \sqrt{|g|}\left[g_{a} g^{c g} g^{d h}\left(\mathcal{K}^{a}{ }_{i c d} \breve{\mathcal{K}}_{j g h}+\mathcal{K}^{a}{ }_{c d i} \breve{\mathcal{K}}_{g h j}+\mathcal{K}^{a}{ }_{d i c} \breve{\mathcal{K}}_{h j g}\right)-\frac{1}{2(\mathrm{~d}+1)} g_{i j} \partial_{a}\left(g^{b f} g^{c g} g^{d h} \mathcal{K}^{a}{ }_{b c d} \breve{\mathcal{K}}_{f g h}\right)\right]\left(\delta g^{i j}\right) .
\end{aligned}
$$

Putting this all together defines the TW energy-momentum tensor $\Theta_{i j}^{\mathrm{S}}$ as

$$
\begin{aligned}
\Theta_{m n}^{\mathrm{S}}= & -\frac{\mathrm{d}-1}{2 \kappa_{0}} \mathcal{P}_{(m n)}+g_{m n}\left[2 J_{0} c \lambda_{0}^{2}\left(-\frac{1}{\mathrm{~d}+1} \nabla_{a}+\Delta_{a}\right) \mathcal{K}^{a}{ }_{b c d} K^{b c d}-\mathcal{L}_{\mathrm{S}}\right] \\
& +2 J_{0} c \lambda_{0}^{2}\left(K_{m c d} K_{n}{ }^{c d}+2 K_{b c m} K^{b c}{ }_{n}\right)+2 J_{0} c\left(8 \mathcal{K}_{m b} \mathcal{K}^{b}{ }_{n}-2 \mathcal{K} \mathcal{K}_{m n}\right) \\
& +2 J_{0} c\left(\mathcal{K}_{m b c d} \mathcal{K}_{n}{ }^{b c d}-\mathcal{K}^{a}{ }_{m c d} \mathcal{K}_{a n}{ }^{c d}-2 \mathcal{K}^{a b c}{ }_{m} \mathcal{K}_{a b c n}\right)
\end{aligned}
$$


where $K_{b c d}=g_{a} \mathcal{K}^{a}{ }_{b c d}+\breve{\mathcal{K}}_{b c d}$ and $\Delta_{a} \equiv g_{a}-\alpha_{a}$ are tensors. This demonstrates that the energy-momentum tensor is indeed manifestly tensorial on $\mathcal{M}$. Since we have used the Gauss-Bonnet action to describe dynamics for $\mathcal{D}_{a b}$, the field equations are second-order differential equations in $g_{a b}$.

\section{Palatini field and metric compatible connection}

It may be convenient to solve the field equations with a connection that is the Levi-Civita connection of a metric. Here we demonstrate how we may exchange the field degrees of $\Pi_{b c}^{a}$ for a tensor, $C^{a}{ }_{b c}$ which we will call the Palatini field, and a Levi-Civita connection associated with the metric $g_{a b}$. Given any two connections, say $\Gamma_{b c}^{a}$ and $\hat{\Gamma}^{a}{ }_{b c}$, their difference is always a tensor. Define $\hat{\Gamma}^{a}{ }_{b c}$ as the Levi-Civita connection associated with $g_{a b}$ so that $\hat{\nabla}_{a} g_{b c}=0$. Then, a Palatini field $C^{a}{ }_{b c}$, can be defined relative to this Levi-Civita connection for any connection $\Gamma_{b c}^{a}$ as

$$
\Gamma_{b c}^{a}=\hat{\Gamma}_{b c}^{a}+C_{b c}^{a} .
$$

Here $C^{a}{ }_{b c}=C^{a}{ }_{c b}$ as there is no torsion. Similarly for the projective invariant, $\Pi_{b c}^{a}$, we may write

$$
\Pi_{b c}^{a}=\hat{\Pi}_{b c}^{a}+\tilde{C}_{b c}^{a},
$$

where $\hat{\Pi}_{b c}^{a}$ is the projective invariant for the equivalence class in which $\hat{\Gamma}_{b c}^{a}$ is a member and $\tilde{C}_{b c}^{a} \equiv C_{b c}^{a}-$ $\frac{1}{\mathrm{~d}+1}\left(\delta^{a}{ }_{c} C_{b}+\delta^{a}{ }_{b} C_{c}\right)$, is trace-free and symmetric in its last two indices. Here $C_{b} \equiv C^{a}$ ba.

Using the Palatini field and the Levi-Civita connection, the Riemann curvature tensor for $\Gamma_{b c}^{a}$ may be written as

$$
\begin{aligned}
R^{m}{ }_{n a b}= & \hat{R}^{m}{ }_{n a b}+\hat{\nabla}_{a} C^{m}{ }_{n b}-\hat{\nabla}_{b} C^{m}{ }_{n a} \\
& +C^{r}{ }_{n b} C^{m}{ }_{a r}-C^{r}{ }_{n a} C^{m}{ }_{b r} \\
\equiv & \hat{R}^{m}{ }_{n a b}+Q^{m}{ }_{n a b} .
\end{aligned}
$$

Similarly,

$$
R_{a b}=\hat{R}_{a b}+Q_{a b},
$$

where $Q_{a b} \equiv Q^{m}{ }_{a m b}$ and $Q=g^{a b} Q_{a b}$. Then the LHS of Eq. (183) may be written as

$$
\frac{1}{2} R_{(a b)}-\frac{1}{2} R g_{a b}=\hat{R}_{a b}-\frac{1}{2} \hat{R} g_{a b}+\frac{1}{2} Q_{(a b)}-\frac{1}{2} Q g_{a b} .
$$

In leu of the field variables $\left\{g_{a b}, \Pi^{r}{ }_{n a}, \mathcal{D}_{a b}\right\}$, the field equations may now be solved using the fields $\left\{g_{a b}, C^{r}{ }_{n a}, \mathcal{D}_{a b}\right\}$ and Eq. (183) becomes
$\hat{R}_{p q}-\frac{1}{2} \hat{R} g_{p q}=\kappa_{0}\left(\Theta_{p q}^{\mathrm{S}}+\Theta_{p q}^{\mathrm{matter}}\right)+\frac{1}{2} Q g_{p q}-\frac{1}{2} Q_{(p q)}$.

In this way, both sides of Eq. (197) are separately divergence-free with respect to the Levi-Civita connection. One sees that both $C^{r}{ }_{n b}$ and $\mathcal{D}_{a b}$ act as geometric sources for the metric compatible Riemannian geometry in general relativity. When $\mathcal{P}_{a b}=0$ and $C^{r}{ }_{n b}=0$ this becomes the usual theory of general relativity. Note in this case, Eq. (53) becomes

$$
\mathcal{D}_{b c}=\partial_{b} g_{c}-\hat{\Gamma}^{e}{ }_{b c} g_{e}-g_{b} g_{c},
$$

which can be eliminated by a choice of coordinates (volume preserving). The analogy of $\mathcal{D}_{a b}$ with vector potentials $A_{a}$ in Yang-Mills theories $[16,19,43,44]$ demonstrates that general relativity is in the "pure gauge" sector of TW gravity. This strategy facilitates finding out whether there are projective geometric contributions to, for example, primordial perfect fluids, the origin of an inflaton and dark matter sources that may not have arisen from the matter Lagrangian. For solutions associated with definite symmetries, one can choose an ansatz for $C^{a}{ }_{b c}$ and $\mathcal{D}_{a b}$ whose Lie derivative with respect to the Killing vectors of the metric vanish. Recent work $[25,29]$ has already shown that projective geometry serves as a source for the cosmological constant. Other issues related to the principle of equivalence, cosmology, holonomy and projectively equivalent manifolds have been studied as well $[7,9,10]$.

\section{GEODESIC DEVIATION}

To complete this study of the gauge covariant field equations and gauge invariant action we examine the geodesic deviation equations on the Thomas cone and their image on the manifold $\mathcal{M}$. Not only does geodesic deviation have importance in tidal forces, it can also provide a mechanism to study radiative degrees of freedom in $\mathcal{D}_{a b}$. Here, we will examine the modification to geodesic deviation that results from the presence of the projective gauge field $\mathcal{D}_{b c}$. A review of geodesic deviation and its derivation in general relativity can be found in textbooks such as [45].

\section{A. The geodesic deviation equation}

Let $\mathcal{M}$ be the spacetime manifold equipped with a metric $g_{a b}$. Recall the geodesic equation for any connection $\Gamma^{a}{ }_{b c}$ on $\mathcal{M}$

$$
\frac{d^{2} x^{a}}{d \tau^{2}}+\Gamma_{b c}^{a} \frac{d x^{b}}{d \tau} \frac{d x^{c}}{d \tau}=f(\tau) \frac{d x^{a}}{d \tau},
$$

where $\tau$ is some parameter. Here $f(\tau)=0$ if and only if $\tau$ is an affine parameter for $\Gamma_{b c}^{a}$. In the presence of a 
gravitational field where the connection $\Gamma_{b c}^{a}$ is compatible with the metric, freely moving objects will travel along geodesics specified by Eq. (199).

Consider the space of geodesics $x^{a}(s, \tau)$, where for each fixed value $s=s_{0}$, we have that $x^{a}\left(s_{0}, \tau\right)$ is a geodesic with affine parameter $\tau$. This gives us a one-parameter family of geodesics which allows us to examine geodesics that are close to each other. The geodesic tangent vector $T^{a}(s, \tau)$ and geodesic deviation vector $X^{a}(s, \tau)$ are given by

$$
\begin{aligned}
T^{a}(s, \tau) & =\frac{\partial x^{a}(s, \tau)}{\partial \tau}, \\
X^{a}(s, \tau) & =\frac{\partial x^{a}(s, \tau)}{\partial s} .
\end{aligned}
$$

Equation (200) leads to an immediate relation between derivatives of $T^{a}$ and $X^{a}$

$$
\frac{\partial X^{a}}{\partial \tau}=\frac{\partial T^{a}}{\partial s} .
$$

For a vector field $V^{a}$ on $\mathcal{M}$, the intrinsic derivative of $V^{a}$ along a curve $x^{a}(\tau)$ is given by

$$
\frac{D V^{a}}{d \tau}=\nabla_{T^{b} \frac{\partial}{\partial x^{b}}} V^{a}=T^{b} \cdot \nabla_{b} V^{a} .
$$

Using Eq. (202), we can find an acceleration by taking the second intrinsic derivative of a vector field. If we do this with the geodesic deviation vector $X^{a}(s, \tau)$ with respect to $\tau$, we find

$$
\begin{aligned}
\frac{D^{2} X^{a}}{\partial \tau^{2}}= & T^{c} \nabla_{c}\left(T^{b} \nabla_{b} X^{a}\right) \\
= & \frac{\partial^{2} T^{a}}{\partial s \partial \tau}+\left(\partial_{c} \Gamma^{a}{ }_{b d}\right) T^{c} T^{b} X^{d} \\
& +\Gamma^{a}{ }_{b d}\left(\frac{\partial T^{b}}{\partial \tau} X^{d}+T^{b} \frac{\partial X^{d}}{\partial \tau}\right) \\
& +\Gamma^{a}{ }_{c d}\left(\frac{\partial T^{d}}{\partial \tau}+\Gamma_{b e}^{d} T^{b} X^{e}\right) T^{c} .
\end{aligned}
$$

Equation (203) can be simplified since $x^{a}(s, \tau)$ is a geodesic curve for all fixed $s$. Due to this fact, we know that

$$
\begin{aligned}
T^{b} \nabla_{b} T^{a} & =0 \\
\Rightarrow X^{c} \nabla_{c}\left(T^{b} \nabla_{b} T^{a}\right) & =0 .
\end{aligned}
$$

Expanding Eq. (204) and rearranging terms yields

$$
\begin{aligned}
\frac{\partial^{2} T^{a}}{\partial s \partial \tau}= & -\left(\partial_{d} \Gamma^{a}{ }_{c b}\right) T^{c} T^{b} X^{d}-\Gamma_{b d}^{a}\left(\frac{\partial T^{b}}{\partial s} T^{d}+\frac{\partial T^{d}}{\partial s} T^{b}\right) \\
& -\Gamma^{a}{ }_{c d}\left(\frac{\partial T^{d}}{\partial \tau}+\Gamma^{d}{ }_{b e} T^{b} T^{e}\right) X^{c} .
\end{aligned}
$$

Using Eq. (205), we eliminate $\frac{\partial^{2} T^{a}}{\partial s \partial \tau}$ from Eq. (203) and find

$$
\begin{aligned}
\frac{D^{2} X^{a}}{\partial \tau^{2}} & =\left(\partial_{c} \Gamma_{d b}^{a}-\partial_{d} \Gamma_{c b}^{a}+\Gamma_{c e}^{a} \Gamma_{d b}^{e}-\Gamma_{b e}^{a} \Gamma_{c b}^{e}\right) T^{c} T^{b} X^{d} \\
& =R_{b c d}^{a} T^{b} T^{c} X^{d} .
\end{aligned}
$$

This is the geodesic deviation equation. Note we did not use metric compatibility to arrive at this expression. The full Riemann curvature tensor $R_{b c d}^{a}$ appears in the geodesic deviation equation, including the Weyl term which does not usually appear in Einstein field equations. Gravitational radiation can influence geodesic deviation directly making it a useful observational tool. We will now explore the projective modifications of the geodesic deviation equation and insights on how the diffeomorphism field may be observed.

\section{B. Projective geodesic deviation}

We turn our attention to the diffeomorphism field which we also may consider as the projective gauge field $\mathcal{D}_{b c}$. To compute the resulting geodesic deviation on the spacetime manifold $\mathcal{M}$ for a general connection, we first must find the geodesic deviation of the TW connection on $\mathcal{N}$, and project this deviation down onto $\mathcal{M}$.

From Eq. (206), the geodesic deviation $X^{\alpha}(\tau)$ of the TW connection on $\mathcal{N}$ is given by

$$
\frac{D^{2} X^{\alpha}}{d \tau^{2}}=\mathcal{K}^{\alpha}{ }_{\beta \sigma \rho} \frac{d x^{\beta}}{d \tau} \frac{d x^{\sigma}}{d \tau} X^{\rho},
$$

where the Greek indices range over all coordinates on $\mathcal{N}$. Now, as in Eq. (59) let

$$
X^{\alpha}=\left(X^{a},-\lambda X^{a} g_{a}+X^{5}\right)
$$

define the projective geodesic deviation vector. We have included a perpendicular component as physical vectors such as $X^{\alpha}=\Psi \tilde{\gamma}^{\alpha} \Psi$ might arise. However, for simplicity we will ignore the $X^{5}$ component in this discussion. We have used $g_{a}$ defined via a metric on $\mathcal{N}$ so as not to spoil the projective covariance of the equation. Let us first consider the geodesic deviation $X^{a}$ where $a$ is a spacetime manifold coordinate specifically (not $\lambda$ ). Since the only nonvanishing components of $\mathcal{K}^{\alpha}{ }_{\beta \sigma \rho}$ are the components $\mathcal{K}^{\lambda}{ }_{b c d}$ and $\mathcal{K}^{a}{ }_{b c d}$, then Eq. (207) reduces for $\alpha=a$ to

$$
\begin{aligned}
\frac{D^{2} X^{a}}{d \tau^{2}} & =K^{a}{ }_{b c d} \frac{d x^{b}}{d \tau} \frac{d x^{c}}{d \tau} X^{d} \\
& =\left(\mathcal{R}^{a}{ }_{b c d}+\delta^{a}{ }_{[c} \mathcal{D}_{d] b}\right) \frac{d x^{b}}{d \tau} \frac{d x^{c}}{d \tau} X^{d} \\
& =\left(R_{b c d}^{a}+\delta^{a}{ }_{[c} \mathcal{P}_{d] b}-\delta^{a}{ }_{b} \mathcal{P}_{[c d]}\right) \frac{d x^{b}}{d \tau} \frac{d x^{c}}{d \tau} X^{d} .
\end{aligned}
$$


Here $R_{b c d}^{a}$ is the Riemann curvature tensor for a connection $\Gamma_{b c}^{a}$ which is not necessarily compatible with the metric defining $g_{a}$. Now the parameter $\tau$ is an affine parameter for the $T W$ connection on $\mathcal{N}$, not for the $\Gamma^{a}{ }_{b c}$ connection on $\mathcal{M}$. If we make a change of parametrization $\tau \rightarrow u$ so that $u$ is an affine parameter for the spacetime manifold connection, we get using Eq. (42)

$$
\begin{aligned}
& \frac{D^{2} X^{a}}{d u}-\left(\mathcal{R}_{b c d}^{a}+\delta^{a}{ }_{[c} \mathcal{D}_{d] b}\right) \frac{d x^{b}}{d u} \frac{d x^{c}}{d u} X^{d}=\left(\frac{2}{\lambda} \frac{d \lambda}{d u}\right) \frac{D X^{a}}{d u} \\
& \Rightarrow \frac{D^{2} X^{a}}{d u}-\left(R_{b c d}^{a}+\delta^{a}{ }_{[c} \mathcal{P}_{d] b}-\delta^{a}{ }_{b} \mathcal{P}_{[c d]}\right) \frac{d x^{b}}{d u} \frac{d x^{c}}{d u} X^{d} \\
& \quad=\left(\frac{2}{\lambda} \frac{d \lambda}{d u}\right) \frac{D X^{a}}{d u} .
\end{aligned}
$$

If we consider the $\lambda$ component, we find

$$
\begin{aligned}
\frac{D^{2} X^{\lambda}}{d \tau^{2}}= & K_{b c d}^{\lambda} \frac{d x^{b}}{d \tau} \frac{d x^{c}}{d \tau} X^{d} \\
\frac{D^{2}\left(-\lambda X^{a} g_{a}\right)}{d \tau^{2}}= & \left(\partial_{[b} \mathcal{D}_{c] a}+\Pi_{a[c}^{d} \mathcal{D}_{b] d}\right) \frac{d x^{b}}{d \tau} \frac{d x^{c}}{d \tau} X^{d} \\
= & \left(\nabla_{[c} \mathcal{P}_{d] b}+\alpha_{[d} \mathcal{P}_{c] b}+\alpha_{[b} \mathcal{P}_{c] d}-R_{b c d}^{a} \alpha^{a}\right) \\
& \times \frac{d x^{b}}{d \tau} \frac{d x^{c}}{d \tau} X^{d} .
\end{aligned}
$$

If we take $g_{a}=0$ as a gauge choice, the left-hand side of the above expression vanishes, leaving

$$
\left(\nabla_{[c} \mathcal{P}_{d] b}+\alpha_{[d} \mathcal{P}_{c] b}+\alpha_{[b} \mathcal{P}_{c] d}-R_{b c d}^{a} \alpha^{a}\right) \frac{d x^{b}}{d \tau} \frac{d x^{c}}{d \tau} X^{d}=0
$$

This illustrates the complexity of $\mathcal{D}_{b c}$ (or equivalently, $\mathcal{P}_{b c}$ ) as a dynamical field, since it has its own field equations and energy-momentum tensor. $\mathcal{D}_{b c}$ will interact with the spacetime geometry and have an effect on $\mathcal{R}^{a}{ }_{b c d}$. Thus geodesic deviation is a valuable resource for observation, and the projective gauge field could explain defects in these observations via Eq. (209).

\section{CONCLUSION}

String theory may be thought of as originating from regulating Feynman diagrams in gravitational theories, by adding a tiny dimension to the point particle as initial data. This regulator quickly takes on a life of its own through the Virasoro algebra, which maintains the reparametrization invariance. It has been shown [20] that a projective structure and subsequent projective geometry are the ubiquitous concepts that give meaning to this reparametrization in any dimension. In projective geometry, a manifold is geometrically classified in terms of its family of geodesics. In many ways, geodesics are the most experimentally available geometric structures that give physicists access to the underlying geometry of a manifold. Affine geodesic lines, whether spacelike, timelike, or even null, enjoy reparametrization invariance irrespective of the underlying metric. Furthermore, the correspondence between the Virasoro algebra and projective geometry is analogous to the correspondence of an affine Lie algebra (a class of Kac-Moody algebras) for one-dimensional gauge transformations to Yang-Mills vector potentials in higher dimensional field theories; see Table I.

The projective geometry of Thomas and later Whitehead [4-6] allows us to form a gauge theory for unparametrized paths which induces a dynamical field called the diffeomorphism field. These projective connections get their dynamics from the Thomas-Whitehead gravitational action defined in [20]. However, those and subsequent results [29] used specific coordinates such as constant volume coordinates and background metrics. In this paper, we present the full gauge invariant Thomas-Whitehead action. There are many advantages of having a gauge invariant theory, including the understanding of spontaneously broken symmetry and the constraints that arise in classical and quantum field theories. The results here show precisely how any Dirac fermion will interact with the diffeomorphism field and how chiral masses become manifest due to a volume scale. These gravitationally induced chiral masses are affected by the dimension of the manifold, the number of gauge fields and the spinor's tensor density.

The use of geodesics extends far beyond gravitational theories and these results may be of value in fluid dynamics, optimization, other gauge theories and even quantum computing. Several projects applying the general

TABLE I. Correspondence of symmetries in string theories to connections in field theories coadjoint elements of the Virasoro algebra, $(B, q)$, consists of a quadratic differential $B$ and a central element $q$. They are in correspondence with the projective connection components $\mathcal{D}_{a b}$ that appear in the projective covariant derivative $\tilde{\nabla}_{\alpha}$. Analogously, the coadjoint elements of the affine Lie algebra (Kac-Moody algebra), $(A, \alpha)$, consisting of a one form $A$ and a central element $\alpha$, are in correspondence with the Yang-Mills connection, $A_{a}$ that appears in the gauge covariant derivative $D_{a}$.

\begin{tabular}{lcccc}
\hline \hline Symmetry & \multicolumn{2}{c}{ String theory } & Field theory \\
\hline Reparametrization Invariance & Algebra: Virasoro & Coadjoint elements: $(B, q)$ & Connection: projective & $\tilde{\nabla}_{\alpha}\left(\mathcal{D}_{b c}, \Pi^{a}{ }_{b c}\right)$ \\
Gauge invariance & Algebra: affine Lie & Coadjoint elements: $(A, \alpha)$ & Connection: Yang-Mills & $D_{a}\left(A_{b}\right)$ \\
\hline \hline
\end{tabular}


TW theory presented in this paper are currently underway including the quantization of the fully covariant TW theory, sourcing of cosmological inflation, constraints imposed by affects on gravitational radiation, and applications to the understanding of dark matter.

\section{ACKNOWLEDGMENTS}

The research of K.S. is supported in part by the endowment of the Ford Foundation Professorship of Physics at Brown University. We thank the Nuclear and Particle Theory Group at the University and especially Patrick Vecera. K.S. would like to thank Kevin Iga and Konstantinos Koutrolikos for helpful discussions. V. R. thanks S. L. Gallon for discussions. The research of K. H. and S. B. is supported by fellowships from the Graduate College at The University of Iowa.

\section{APPENDIX A: UNITS, CONVENTIONS, AND HELPFUL CALCULATIONS}

The units of the various constants used throughout this paper for $\mathrm{d}=4$ are

$$
\begin{aligned}
{\left[J_{0}\right] } & =\frac{M L^{2}}{T}, \quad\left[\mathcal{D}_{a b}\right]=\left[R_{a b}\right]=L^{-2}, \\
{[\ell] } & =\text { dimensionless, } \quad\left[\kappa_{0}\right]=\frac{T^{2}}{M L} \\
{\left[d^{\mathrm{d}} x\right] } & =T L^{\mathrm{d}-1} .
\end{aligned}
$$

We may at times set $c=1$ but expose factors of $c$ when calculating numerical values. Latin indices take values $a, b, \ldots=0,1,2, \ldots, \mathrm{d}-1$ and Greek indices take values $\mu, \nu, \ldots=0,1,2, \ldots, \mathrm{d}$, with the exception of the Greek letter $\lambda$, which refers to the projective coordinate $x^{\mathrm{d}}=\lambda=\lambda_{0} \ell$. A coordinate transformation and corresponding Jacobian matrix over the d-dimensional space is given as

$$
x^{\prime m}=x^{\prime m}\left(x^{n}\right), \quad J_{n}^{m}=\frac{\partial x^{\prime m}}{\partial x^{n}} .
$$

A useful property of the determinant of the Jacobian matrix is its derivative in terms of the coordinates:

$$
\frac{\partial \log |J|}{\partial x^{a}}=\frac{\partial x^{n}}{\partial x^{\prime m}} \frac{\partial}{\partial x^{a}} \frac{\partial x^{\prime m}}{\partial x^{n}} .
$$

Our conventions for the Riemann curvature tensor $R^{a}{ }_{b c d}$ are the same as for the projective curvature $\mathcal{K}^{\mu}{ }_{\nu \alpha \beta}$. The Riemann curvature tensor is written in terms of $\Gamma^{m}{ }_{a b}$ where as the projective curvature is written in terms of $\tilde{\Gamma}_{\alpha \beta}^{\mu}$ :

$$
\mathcal{K}_{\nu \alpha \beta}^{\mu} \equiv \tilde{\Gamma}_{\nu[\beta, \alpha]}^{\mu}+\tilde{\Gamma}_{\nu[\beta}^{\rho} \tilde{\Gamma}_{\alpha] \rho}^{\mu} .
$$

Here and throughout, brackets mean antisymmetrization and parentheses mean symmetrization
$\mathcal{K}_{\beta[\mu \nu]}^{\alpha}=\mathcal{K}_{\beta \mu \nu}^{\alpha}-\mathcal{K}_{\beta \nu \mu}^{\alpha}, \quad \mathcal{K}_{(\mu \nu)}=\mathcal{K}_{\mu \nu}+\mathcal{K}_{\nu \mu}$.

Equation (A4) means the following must be true:

$$
\begin{gathered}
{\left[\tilde{\nabla}_{\alpha}, \tilde{\nabla}_{\beta}\right] V^{\gamma}=\mathcal{K}_{\rho \alpha \beta}^{\gamma} V^{\rho}} \\
{\left[\tilde{\nabla}_{\alpha}, \tilde{\nabla}_{\beta}\right] V_{\gamma}=-\mathcal{K}_{\gamma \alpha \beta}^{\rho} V_{\rho} .}
\end{gathered}
$$

The d-dimensional metric $g_{a b}$ is promoted to the Thomas cone metric $G_{\alpha \beta}$ by adding the appropriate projective contributions to the components. An easy way to see this is by writing

$$
G_{\alpha \beta}=\left[\begin{array}{cc}
g_{a b}-\lambda_{0}^{2} g_{a} g_{b} & -\frac{\lambda_{0}{ }^{2}}{\lambda} g_{a} \\
-\frac{\lambda_{0}{ }^{2}}{\lambda} g_{b} & -\frac{\lambda_{0}{ }^{2}}{\lambda^{2}}
\end{array}\right],
$$

$$
\begin{gathered}
G^{\alpha \beta}=\left[\begin{array}{cc}
g^{a b} & -\lambda g^{a m} g_{m} \\
-\lambda g^{b m} g_{m} & \frac{\lambda^{2}}{\lambda_{0}^{2}}\left(-1+g^{m n} \lambda_{0}{ }^{2} g_{m} g_{n}\right)
\end{array}\right], \\
G_{\alpha \beta}=\delta^{a}{ }_{\alpha} \delta^{b}{ }_{\beta} g_{a b}-\lambda_{0}^{2} g_{\alpha} g_{\beta},
\end{gathered}
$$

$G^{\alpha \beta}=g^{a b}\left(\delta^{\alpha}{ }_{a}-g_{a} \Upsilon^{\alpha}\right)\left(\delta^{\beta}{ }_{b}-g_{b} \Upsilon^{\beta}\right)-\lambda_{0}^{-2} \Upsilon^{\alpha} \Upsilon^{\beta}$

where the d-dimensional metric $g_{a b}$ has signature $(+,-,-,-, \cdots,-)$ and the dimensionless parameter $\ell=\lambda / \lambda_{0}$. The function $g_{a} \equiv \frac{1}{d+1} \partial_{a} \log \sqrt{|g|}$ is chosen as it transforms like the trace of a connection and depends only on the metrics determinant. The d-dimensional Riemann curvature tensor $R_{b c d}^{a}$ satisfies the same relation as the $(\mathrm{d}+1)$-dimensional tensor $\mathcal{K}^{\alpha}{ }_{\beta \mu \nu}$, Eq. (III C), but in terms of the d-dimensional covariant derivative $\nabla_{a}$. The commutator of covariant derivatives on an arbitrary rank $m$ covariant, rank $n$-contravariant tensor is equivalent to the following action of $R_{b c d}^{a}$ :

$$
\begin{aligned}
{\left[\nabla_{a},\right.} & \left.\nabla_{b}\right] T_{c_{1} \ldots c_{m}}{ }^{d_{1} \ldots d_{n}} \\
= & -R^{e}{ }_{c_{1} a b} T_{e c_{2} \ldots c_{m}}{ }^{d_{1} d_{2} \ldots d_{n}}-\cdots-R^{e}{ }_{c_{m} a b} T_{c_{1} c_{2} \ldots e} d_{1} d_{2} \ldots d_{n} \\
& +R^{d_{1}}{ }_{e a b} T_{c_{1} \ldots c_{m}}{ } \ldots d_{n}+\cdots+R^{d_{m}} e_{e a b} T_{c_{1} \ldots c_{m}} d_{1} \ldots e
\end{aligned}
$$

We list all nonvanishing connections and curvatures below:

$$
\begin{gathered}
\tilde{\Gamma}_{b c}^{a}=\Pi_{b c}^{a} ; \quad \tilde{\Gamma}_{a b}^{\lambda}=\lambda \mathcal{D}_{a b}, \\
\tilde{\Gamma}^{a}{ }_{\lambda b}=\tilde{\Gamma}_{b \lambda}^{a}=\lambda^{-1} \delta_{b}^{a}, \\
\Pi_{b c}^{a}=\Gamma_{b c}^{a}+\delta_{(c}^{a} \alpha_{b)}, \\
\mathcal{P}_{b c}=\mathcal{D}_{b c}-\partial_{b} \alpha_{c}+\Gamma_{b c}^{e} \alpha_{e}+\alpha_{b} \alpha_{c}, \\
\mathcal{K}_{b c d}^{a}=R_{b c d}^{a}+\delta^{a}{ }_{[c} \mathcal{P}_{d] b}-\delta_{b}^{a} \mathcal{P}_{[c d]},
\end{gathered}
$$




$$
\begin{aligned}
& \mathcal{K}^{a}{ }_{b c d}=\mathcal{R}^{a}{ }_{b c d}+\delta^{a}{ }_{[c} \mathcal{D}_{d] b}, \\
& \mathcal{K}^{\lambda}{ }_{c a b}=\lambda \partial_{[a} \mathcal{D}_{b] c}+\lambda \Pi_{c[b}^{d} \mathcal{D}_{a] d}, \\
& \mathcal{K}_{b c d}^{\lambda}=\lambda\left(\partial_{[c} \mathcal{P}_{d] b}+\Gamma_{b[d}^{a} \mathcal{P}_{c] a}+\alpha_{[d} \mathcal{P}_{c] b}\right. \\
& \left.+\alpha_{b} \mathcal{P}_{[c d]}-R_{b c d}^{a} \alpha_{a}\right), \\
& \breve{\mathcal{K}}_{b c d} \equiv \frac{1}{\lambda} \mathcal{K}_{b c d}^{\lambda}, \\
& \mathcal{K}^{\mu}{ }_{b \mu d}=\mathcal{K}_{b d}=\mathcal{R}_{b d}+(\mathrm{d}-1) \mathcal{D}_{b d} \\
& =R_{b d}+\mathrm{d} \mathcal{P}_{d b}-\mathcal{P}_{b d}, \\
& \mathcal{K} \equiv G^{\alpha \beta} \mathcal{K}_{\alpha \beta}=\mathcal{R}+(\mathrm{d}-1) \mathcal{D}=R+(\mathrm{d}-1) \mathcal{P}, \\
& R=g^{a b} R_{a b}, \quad \mathcal{P}=g^{a b} \mathcal{P}_{a b}, \\
& K_{b c d} \equiv g_{a} \mathcal{K}^{a}{ }_{b c d}+\breve{\mathcal{K}}_{b c d} \\
& =\left(g_{a}-\alpha_{a}\right) R_{b c d}^{a}+\left(g_{c}-\alpha_{c}\right) \mathcal{P}_{d b}-\left(g_{d}-\alpha_{d}\right) \mathcal{P}_{c b} \\
& -\left(g_{b}-\alpha_{b}\right) \mathcal{P}_{[c d]}+\nabla_{c} \mathcal{P}_{d b}-\nabla_{d} \mathcal{P}_{c b}, \\
& \hat{\mathcal{K}}_{a}{ }^{b g r}=\mathcal{K}^{\bar{a}}{ }_{\bar{b} \bar{g} \bar{r}} \mathcal{G}_{a \bar{a}}{ }^{b \bar{b}}[\bar{g}|\bar{g}| r] \bar{r}, \\
& +\delta^{\gamma}{ }_{\alpha} \delta^{\bar{\gamma}}{ }_{\bar{\alpha}}^{\beta \rho} G^{\bar{\beta} \bar{\rho}} \text {. }
\end{aligned}
$$

\section{APPENDIX B: FIELD EQUATIONS IN THE ABSENCE OF MATTER LAGRANGIANS}

The field equations for $\Pi_{b c}^{a}$ read as

$$
\begin{gathered}
E_{a}{ }^{m n}-\frac{1}{\mathrm{~d}+1} \delta_{a}{ }^{(m} E_{b}{ }^{n) b}=0, \\
E_{a}{ }^{m n}=E_{a}{ }^{n m} \\
=\frac{1}{2 \kappa_{0} J_{0} c} \breve{\nabla}_{a}\left(\sqrt{|g|} g^{m n}\right)-\breve{\nabla}_{c}\left(\sqrt{|g|} \hat{\mathcal{K}}_{a}^{(m n) c}\right) \\
+2 \lambda_{0}^{2} \breve{\nabla}_{c}\left(\sqrt{|g|} g_{a} \breve{\mathcal{K}}^{(m n) c}\right)-2 \lambda_{0}^{2} \sqrt{|g|} K^{(m n) c} \mathcal{D}_{c a} .
\end{gathered}
$$

The field equations for $\mathcal{D}_{a b}$ read as

$$
\begin{aligned}
& -\frac{1}{2 \kappa_{0} J_{0} c} \sqrt{|g|}(\mathrm{d}-1) g^{p q}+\sqrt{|g|} \hat{\mathcal{K}}_{c}{ }^{(p q) c} \\
& \quad+2 \lambda_{0}^{2} \breve{\nabla}_{g}\left(\sqrt{|g|} K^{(p q) g}\right)-2 \lambda_{0}^{2} \sqrt{|g|} g_{c} \breve{\mathcal{K}}^{(p q) c}=0 .
\end{aligned}
$$

The field equations for $g_{a b}$ read as

$$
\frac{1}{2} R_{(p q)}-\frac{1}{2} R g_{p q}=\kappa_{0} \Theta_{p q}^{\mathrm{S}}
$$

$$
\begin{aligned}
\Theta_{m n}^{\mathrm{S}}= & -\frac{\mathrm{d}-1}{2 \kappa_{0}} \mathcal{P}_{(m n)}+g_{m n}\left[2 J_{0} c \lambda_{0}^{2}\left(-\frac{1}{\mathrm{~d}+1} \nabla_{a}+\Delta_{a}\right) \mathcal{K}^{a}{ }_{b c d} K^{b c d}-\mathcal{L}_{\mathrm{S}}\right] \\
& +2 J_{0} c \lambda_{0}^{2}\left(K_{m c d} K_{n}{ }^{c d}+2 K_{b c m} K^{b c}{ }_{n}\right)+2 J_{0} c\left(8 \mathcal{K}_{m b} \mathcal{K}^{b}{ }_{n}-2 \mathcal{K} \mathcal{K}_{m n}\right) \\
& +2 J_{0} c\left(\mathcal{K}_{m b c d} \mathcal{K}_{n}{ }^{b c d}-\mathcal{K}^{a}{ }_{m c d} \mathcal{K}_{a n}{ }^{c d}-2 \mathcal{K}^{a b c}{ }_{m} \mathcal{K}_{a b c n}\right) \\
\mathcal{L}_{\mathrm{S}}= & -\frac{1}{2 \kappa_{0}}(\mathrm{~d}-1) \mathcal{P}+c J_{0} \lambda_{0}{ }^{2} K_{b c d} K^{b c d}-c J_{0}\left(\mathcal{K}^{a}{ }_{b c d} \mathcal{K}_{a}{ }^{b c d}-4 \mathcal{K}_{a b} \mathcal{K}^{a b}+\mathcal{K}^{2}\right) .
\end{aligned}
$$

[1] T. Crilly, Historia mathematica 26, 125 (1999).

[2] E. Cartan, Ann. Sci. Ecole Norm. Sup. 40, 325 (1923).

[3] E. Cartan, Bull. Soc. Math. Fr. 2, 205 (1924).

[4] T. Y. Thomas, Proc. Natl. Acad. Sci. U.S.A. 11, 588 (1925).

[5] T. Y. Thomas, Proc. Natl. Acad. Sci. U.S.A. 11, 199 (1925).

[6] J. Whitehead, Ann. Math. 32, 327 (1931).

[7] G. S. Hall and D. P. Lonie, Classical Quant. Grav. 24, 3617 (2007).

[8] G. S. Hall and D. P. Lonie, J. Math. Phys. (N.Y.) 49, 022502 (2008).
[9] G. S. Hall and D. P. Lonie, Classical Quant. Grav. 26, 125009 (2009).

[10] G. S. Hall and D. P. Lonie, Classical Quant. Grav. 28, 083101 (2011).

[11] P. Nurowski, J. Geom. Phys. 62, 657 (2012).

[12] M. Virasoro, Phys. Rev. D 1, 2933 (1970).

[13] A. Pressley and G. Segal, Loop Groups, Oxford Mathematical Monographs (Clarendon, Oxford, 1988).

[14] V. Ovsienko and S. Tabachnikov, Cambridge Tracts in Mathematics (Cambridge University Press, Cambridge, England, 2005), p. 165. 
[15] A. A. Kirillov, Lect. Notes Math. 970, 101 (1982).

[16] B. Rai and V. G. J. Rodgers, Nucl. Phys. B341, 119 (1990).

[17] A. Alekseev and S. L. Shatashvili, Nucl. Phys. B323, 719 (1989).

[18] A. Alekseev, L. D. Faddeev, and S. L. Shatashvili, J. Geom. Phys. 5, 391 (1988).

[19] V. Rodgers, Phys. Lett. B 336, 343 (1994).

[20] S. Brensinger and V. G. J. Rodgers, Int. J. Mod. Phys. A 33, 1850223 (2018).

[21] R. Liouville, C.R. Hebd. Seances Acad. Sci. 105, 1062 (1887), https://archive.org/details/comptesrendusheb1051887acad/page/n7/mode/2up.

[22] R. Liouville, J. Ecole Politech. Cah.59, 7 (1889), https:// babel.hathitrust.org/cgi/pt?id=coo.31924069342339\&view= 1 up\&seq $=595$.

[23] A. Cap, A. R. Gover, and H. R. Macbeth, Geometriae Dedicata 168 (2014).

[24] S. N. Curry and A. R. Gover, in Asymptotic Analysis in General Relativity, London Mathematical Society Lecture Note Series (Cambridge University Press, Cambridge, England, 2018), pp. 86-170.

[25] A. R. Gover and H. Macbeth, Differential Geometry and its Applications 33, 44 (2014).

[26] A. R. Gover, E. Latini, and A. Waldron, Commun. Math. Phys. 341, 667 (2016).

[27] T. Bailey, M. Eastwood, and A. Gover, Rocky Mt. J. Math. 24, 1191 (1994).

[28] M. Eastwood, Math. Appl. 144, 41 (2007).

[29] S. Brensinger, K. Heitritter, V. G. J. Rodgers, K. Stiffler, and C. A. Whiting, Classical Quant. Grav. 37, 055003 (2020).
[30] A. M. Polyakov, Phys. Lett. 103B, 207 (1981).

[31] A. M. Polyakov, Mod. Phys. Lett. A 02, 893 (1987).

[32] A. Palatini, Rend. Circ. Mat. Palermo 43, 203 (1919).

[33] D. Lovelock, J. Math. Phys. (N.Y.) 12, 498 (1971).

[34] Our extra direction $\lambda$ is related to Thomas's original extra direction in [4,5] through an exponential as in [35]. Furthermore, Thomas referred to this extra direction as the 0th direction where we refer to it as the dth direction, reserving the index 0 for time as is common in physics literature.

[35] M. Crampin and D. Saunders, J. Geom. Phys. 57, 691 (2007).

[36] M. Eastwood and V. S. Matveev, Math. Appl. 144, 339 (2007).

[37] C. Roberts, Differential Geometry and its Applications 5, 237 (1995).

[38] S. J. Brensinger, Ph.D. thesis, University of Iowa, 2020.

[39] G. Segal, Commun. Math. Phys. 80, 301 (1981).

[40] E. Witten, Commun. Math. Phys. 114, 1 (1988).

[41] I. M. Gelfand and D. B. Fuchs, Funct. Anal. Appl. 3, 194 (1969).

[42] V. Ogievetsky and I. Polubarinov, Sov. Phys. JETP 21, 1093 (1965).

[43] R. P. Lano and V. G. J. Rodgers, Nucl. Phys. B437, 45 (1995).

[44] V. G. J. Rodgers and T. Yasuda, Mod. Phys. Lett. A 18, 2467 (2003).

[45] M. Hobson, G. Efstathiou, and A. Lasenby, General Relativity: An Introduction for Physicists (Cambridge, University Press, Cambridge, England, 2006). 